\title{
WALL-CROSSING FORMULAS, BOTT RESIDUE FORMULA AND THE DONALDSON INVARIANTS OF RATIONAL SURFACES
}

\author{
GEIR ELLINGSRUD AND LOTHAR GÖTTSCHE
}

\section{INTRODUCTION}

The Donaldson invariants of a smooth 4 manifold $M$ depend by definition on the choice of a Riemannian metric. In case $b^{+}(M)>1$ they however turn out to be independent of the metric as long as it is generic, and thus they give $C^{\infty}$-invariants of $M$. In case $b_{+}(M)=1$ the invariants have been introduced and studied by Kotschick in [Ko]. It turns out that the positive cone of $M$ has a chamber structure, and Kotschick and Morgan show in [K-M that the invariants only depend on the chamber of the period point of the metric.

Now let $S$ be a smooth algebraic surface with geometric genus $p_{g}(S)=0$, irregularity $q(S)=0$, and let $H$ be an ample divisor on $S$. Let $M_{H}^{S}\left(c_{1}, c_{2}\right)$ be the moduli space of $H$-Gieseker semistable rank 2 sheaves on $S$ with Chern classes $c_{1}$ and $c_{2}$. In the recent paper E-G we studied the variation of $M_{H}^{S}\left(c_{1}, c_{2}\right)$ and that of the corresponding Donaldson invariants under change of the ample divisor $H$. For the Donaldson invariants this corresponds to restricting our attention from the positive cone of $S$ to the subcone of ample classes. We imposed a suitable additional condition on the walls between two chambers and called walls satisfying this condition good walls.

We showed that if the polarisation $H$ passes through a good wall $W$ defined by a cohomology class $\xi \in H^{2}(S, \mathbb{Z})$, then $M_{H}^{S}\left(c_{1}, c_{2}\right)$ changes by a number of flips. Following $\mathrm{K}-\mathrm{M}$ we wrote the change of the degree $N$ Donaldson invariant as a sum of contributions $\delta_{\xi, N}$ with $\xi$ running through the set of cohomology classes defining $W$. We then used our flip description to compute $\delta_{\xi, N}$ in terms of Segre classes of certain standard bundles $\mathcal{V}_{\xi, N}$ over a Hilbert scheme of points $\operatorname{Hilb}^{d_{\xi, N}}(S \sqcup S)$ on two disjoint copies of $S$ (here $d_{\xi, N}=\left(N+3+\xi^{2}\right) / 4$ ). We proceeded to compute the leading terms of $\delta_{\xi, N}$ explicitely and formulated a conjecture about the precise shape of $\delta_{\xi, N}$, related to a conjecture from [K-M]. We will in future refer to any formula for $\delta_{\xi, N}$ as a wall-crossing formula.

Most of the results of [E-G] were also obtained independently in [F-Q], and a flip description of the change of the moduli spaces was obtained independently for varieties of arbitrary dimension and sheaves of arbitrary rank in $\mathrm{M}-\mathrm{W}$. In $[\mathrm{H}-\mathrm{P}$ a Feynman path integral aproach to this problem is developed, and some of the leading terms of the wall-crossing formulas are determined.

The current paper is a continuation of [E-G]. We specialize to the case that the surface $S$ is rational. The first advantage is that now almost always all walls are good and so the formulas from E-G almost always apply.

Key words and phrases. Moduli spaces, Donaldson invariants, Hilbert scheme of points. 
The main reason for restricting our attention to rational surfaces is that they allow us to use an additional powerful tool: the Bott residue formula. A rational surface can always be deformed to a surface admitting an action of a two-dimensional algebraic torus $\Gamma$ with only a finite number of fixpoints. As the Donaldson invariants are in particular deformation invariants, we can assume that $S$ admits such an action of $\Gamma$. It is easy to see that this action will lift to the Hilbert schemes $\operatorname{Hilb}^{d_{\xi, N}}(S \sqcup S)$, and that the standard bundles $\mathcal{V}_{\xi, N}$ are equivariant for the induced action. Furthermore also the induced action will only have a finite number of fixpoints, and the same is true for a general 1-parameter subgroup $T$ of $\Gamma$. The weights of the action of $T$ on the tangent spaces of $\operatorname{Hilb}^{d_{\xi, N}}(S \sqcup S)$ and on the fibres of $\mathcal{V}_{\xi, N}$ at the fixpoints can be determined explicitely from the corresponding weights on $S$. So we can apply the Bott residue formula to this situation and, given $N$ and $\xi$ and the weights on $S$, we always have an algorithm to compute the change $\delta_{\xi, N}$ explicitely. This algorithm involves very many computations, so we use a suitable Maple program.

Now let $S$ be a rational ruled surface with projection $t: S \longrightarrow \mathbb{P}_{1}$. Let $F$ be the class of a fibre of $t$ and assume that the intersection number $c_{1} . F$ is 1 . Then [Q2] shows that, given $c_{2} \in H^{2}(S, \mathbb{Z})$,

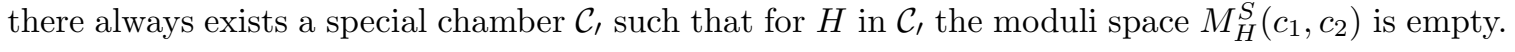
In particular the corresponding $S O(3)$-invariant is zero on $\mathcal{C}_{1}$. This already gives us an algorithm for computing all the $S O(3)$-invariants corresponding to first Chern classes $c_{1}$ with $c_{1} \cdot F=1$ on $S$. Given a chamber $\mathcal{C}$ we obtain the value of the invariant by just summing up all the changes for all the walls between $\mathcal{C}$ and $\mathcal{C}_{\text {, }}$.

At this point we can combine our methods with an additional ingredient: The blowup formulas, which relate the Donaldson invariants of an algebraic surface $S$ with those of the blowup $\widehat{S}$ of $S$ in a point. In the case of the projective plane $\mathbb{P}_{2}$ we obtain an algorithm for computing all the $S O(3)$ and $S U(2)$-invariants. Let $\rho: \widehat{\mathbb{P}}_{2} \longrightarrow \mathbb{P}_{2}$ be the blow up of $\mathbb{P}_{2}$ in a point, let $H, F$ and $E$ be the hyperplane class, the fibre of the projection $\widehat{\mathbb{P}}_{2} \longrightarrow \mathbb{P}_{1}$ and the exceptional divisor respectively. We obtain the $S O(3)$-invariants of $\mathbb{P}_{2}$ by first computing the invariants on $\widehat{\mathbb{P}}_{2}$ corresponding to $c_{1}=\rho^{*}(H)$ and applying the $S U(2)$-blowup formulas. Similarly we obtain the the $S U(2)$-invariants of $\mathbb{P}_{2}$ by first computing those on $\widehat{\mathbb{P}}_{2}$ corresponding to $c_{1}=E$ and applying the $S O(3)$-blowup formulas. Notice that in both cases $c_{1} \cdot F=1$ on $\widehat{\mathbb{P}}_{2}$, so that the algorithm of the previous paragraph applies. Using a suitable Maple program we have computed all the $S O(3)$ - and $S U(2)$-invariants of $\mathbb{P}_{2}$ of degree smaller then 50 .

$S O(3)$ - and $S U(2)$-invariants of $\mathbb{P}_{2}$ and rational ruled surfaces had already been computed by several authors (see e.g. [L-Q] E-LP-S and [K-L]) using a variety of methods. In K-L Kotschick and Lisca have already made use of the blowup formulas in combination with the wall-crossing formulas. Their computations also involve for the first time the 4-dimensional class. Their results agree with ours up to diffenent conventions. Our paper is partially motivated by and built on K-L. In particular we found there the correct formulation and the references for the blowup formulas in the case $b_{+}=1$.

We then go back to the wall-crossing formulas. Assuming the conjecture from [E-G] about the shape of $\delta_{\xi, N}$ we are able to determine (again with a suitable Maple program) the first 5 leading 
terms of $\delta_{\xi, N}$ and using an additional conjecture even the first 7 leading terms. Furthermore, again using the conjecture, we determine $\delta_{\xi, N}$ on a rational ruled surface for $d_{\xi, N} \leq 8$. By explicitely determining the corresponding $\delta_{\xi, N}$ we show that on a rational ruled surface the conjecture and all the formulas are correct for all walls $\xi$ and all $N$ with $N \leq 40$ and $d_{\xi, N} \leq 8$.

Now we compute the Donaldson invariants for rational ruled surfaces $S$ by again combining the wall-crossing formulas with the blowup formulas. We apply this algorithm to compute all the invariants on $S$ of degree at most 35 . The result shows that the special chamber $\mathcal{C}_{\text {, }}$, where the invariants corresponding to first Chern class $c_{1}$ with $c_{1} \cdot F=1$ vanish, is also special for all other $c_{1}$. We obtain that in the chamber $\mathcal{C}$, the Donaldson invariants can be expressed as a polynomial in the linear form $L_{F}$ defined by $F$ and the quadratic form $q_{S}$. This polynomial is independent of $S$, and there is a simple relationship between the polynomials for different $c_{1}$.

Finally we observe that by combining the results obtained so far with the blowup formulas, we obtain an algorithm for computing all the $S O(3)$ and $S U(2)$-invariants for all rational surfaces $S$ for all polarisations in a reasonably big part of the ample cone of $S$. This can be seen as a generalization of the result of [K-L] that the Donaldson invariants of $\mathbb{P}_{2}$ and $\mathbb{P}_{1} \times \mathbb{P}_{1}$ are determined by the wallcrossing formulas on some blowups.

The explicit computations of the wall-crossing formulas and the Donaldson invariants of rational surfaces gives us a lot of empirical data about the shape of these invariants. We have therefore tried to find some patterns in the results and so the paper also contains a number of conjectures and questions. Several of these can already be motivated by the results of K-L.

We would like to thank Dieter Kotschick for sending us the preprint [K-I], which was quite important for our work, and also for some useful comments. Furthermore the second author would like to thank S.A. Strømme for a sample Maple program for computations on Hilbert schemes of points.

\section{BACKGROUND MATERIAL}

In this paper let $S$ be a rational surface over $\mathbb{C}$. For such a surface the natural map from the group of divisors modulo rational equivalence to $H^{2}(S, \mathbb{Z})$ is an isomorphism. So, for $\xi \in H^{2}(S, \mathbb{Z})$, we will often write $\mathcal{O}_{S}(\xi)$ for the line bundle associated to a divisor with class $\xi$.

For a polarization $H$ of $S$ we denote by $M_{H}^{S}\left(c_{1}, c_{2}\right)$ the moduli space of torsion-free sheaves $E$ on $S$ which are H-semistable (in the sense of Gieseker and Maruyama) of rank 2 with $c_{1}(E)=c_{1}$ and $c_{2}(E)=c_{2}$.

Notation 2.1. For a sheaf $\mathcal{F}$ on a scheme $X$ and a divisor $D$ let $\mathcal{F}(D):=\mathcal{F} \otimes \mathcal{O}_{X}(D)$. If $X$ is a smooth variety of dimension $n$, we denote the cup product of two elements $\alpha$ and $\beta$ in $H^{*}(X, \mathbb{Z})$ by $\alpha \cdot \beta$ and the degree of a class $\alpha \in H^{2 n}(X, \mathbb{Z})$ by $\int_{X} \alpha$. For $\alpha, \beta \in H^{2}(S, \mathbb{Z})$ let $\langle\alpha \cdot \beta\rangle:=\int_{S} \alpha \cdot \beta$. We write $\alpha^{2}$ for $\langle\alpha \cdot \alpha\rangle$ and, for $\gamma \in H^{2}(S, \mathbb{Z})$, we put $\langle\alpha, \gamma\rangle:=\langle\alpha \cdot \check{\gamma}\rangle$, where $\check{\gamma}$ is the Poincaré dual of $\gamma$. We denote by $q_{S}$ the quadratic form on $H_{2}(S, \mathbb{Z})$ and, for a class $\eta \in H^{2}(S, \mathbb{Q})$ by $L_{\eta}$ the corresponding linear form on $H_{2}(S, \mathbb{Q})$. 
Convention 2.2. When we are considering surfaces $S$ and $X$ with a morphism $f: X \longrightarrow S$, that is either canonical or clear from the context, then for a cohomology class $\alpha \in H^{*}(Y, \mathbb{Z})$ (or a line bundle $L$ on $Y$ ) we will very often also denote the pull-back via $f$ by $\alpha$ (resp. $L$ ). (Very often $f$ will be a sequence of blowups. In particular if $X$ is a surface which is obtained by $\mathbb{P}_{2}$ by a number of blowups, then we denote by $H$ the pullback of the hyperplane class. Similarly on $\mathbb{P}_{1} \times \mathbb{P}_{1}$ or a variety obtained from $\mathbb{P}_{1} \times \mathbb{P}_{1}$ by a number of blowups, we denote by $F$ and $G$ the classes of the fibres of the projections to the two factors.)

2.1. Walls and chambers. (see [Q1], Q2], Gä], [K-M] and [E-G].)

Definition 2.3. Let $C_{S}$ be the ample cone in $H^{2}(S, \mathbb{R})$. For $\xi \in H^{2}(S, \mathbb{Z})$ let

$$
W^{\xi}:=C_{S} \cap\left\{x \in H^{2}(S, \mathbb{R}) \mid\langle x \cdot \xi\rangle=0\right\} .
$$

We shall call $W^{\xi}$ a wall of type $\left(c_{1}, c_{2}\right)$, and say that it is defined by $\xi$ if the following conditions are satisfied:

1. $\xi+c_{1}$ is divisible by 2 in $N S(S)$,

2. $c_{1}^{2}-4 c_{2} \leq \xi^{2}<0$,

3. there is a polarisation $H$ with $\langle H \cdot \xi\rangle=0$.

In particular $d_{\xi, N}:=\left(4 c_{2}-c_{1}^{2}+\xi^{2}\right) / 4$ is a nonnegative integer. An ample divisor $H$ is said to lie in the wall $W$ if $[H] \in W$. If $D$ is a divisor with $[D]=\xi$, we will also say that $D$ defines the wall $W$.

A chamber of type $\left(c_{1}, c_{2}\right)$ or simply a chamber, is a connected component of the complement of the union of all the walls of type $\left(c_{1}, c_{2}\right)$. We will call a wall $W$ good, if $D+K_{S}$ is not effective for any divisor $D$ defining the wall $W$. If $\left(c_{1}, c_{2}\right)$ are given, we call a polarization $L$ of $S$ generic if it does not lie on a wall of type $\left(c_{1}, c_{2}\right)$.

On a rational surface $S$ we will call a divisor $L$ good if $\left\langle L \cdot K_{S}\right\rangle<0$, and we denote by $C_{S, g}$ the real cone of all good ample divisors. We see that any wall $W$ intersecting $C_{S, g}$ is a good wall.

Let $L_{-}$and $L_{+}$be two divisors on $S$. We denote by $W_{\left(c_{1}, c_{2}\right)}\left(L_{-}, L_{+}\right)$the set of all $\xi \in H^{2}(S, \mathbb{Z})$ defining a wall of type $\left(c_{1}, c_{2}\right)$ and satisfying $\left\langle\xi \cdot L_{-}\right\rangle<0<\left\langle\xi \cdot L_{+}\right\rangle$. We notice that for $L_{-}$and $L_{+}$ good all the walls $W^{\xi}$ defined by $\xi \in W_{\left(c_{1}, c_{2}\right)}\left(L_{-}, L_{+}\right)$are good.

2.2. The change of the Donaldson invariants in terms of Hilbert schemes. In [Ko] the Donaldson invariants have been introduced for 4 -manifolds $M$ with $b_{+}(M)=1$. In [K-M] it has been shown that in case $b_{+}(M)=1, b_{1}(M)=0$ they depend only on the chamber of the period point of the metric in the positive cone of $H^{2}(M, \mathbb{R})$. We want to use conventions from algebraic geometry, which differ by a sign from the usual conventions for Donaldson invariants and furthermore by a factor of a power of 2 from the conventions of [Ka].

Notation 2.4. Let $S$ be a simply connected algebraic surface with $p_{g}(S)=0$. Let $N:=4 c_{2}-c_{1}^{2}-3$ be a nonnegative integer. We denote by $A_{N}(S)$ the set of polynomials of weight $N$ on $H_{2}(S, \mathbb{Q}) \oplus$ $H_{0}(S, \mathbb{Q})$, where we give weight $2-i$ to a class in $H_{2 i}(S, \mathbb{Q})$. Let $\gamma_{c_{1}, N, g}^{S}$ be the Donaldson polynomial 
of degree $N$ with respect to a generic Riemannian metric $g$ associated to the principal $S O(3)$-bundle $P$ on $S$ whose second Stiefel-Whitney class $w_{2}(P)$ is the reduction of $c_{1} \bmod 2$ (in the conventions of e.g. [F-S]). Then $\gamma_{c_{1}, N, g}^{S}$ is a linear map $A_{N}(S) \longrightarrow \mathbb{Q}$. If $N$ is not congruent to $-c_{1}^{2}-3$ modulo 4, then by definition $\gamma_{c_{1}, N, g}^{S}=0$. If $g$ is the Fubini-Studi metric associated to generic ample divisor $L$ on $S$ we denote $\Phi_{c_{1}, N}^{S, L}:=(-1)^{\left(c_{1}^{2}+\left\langle c_{1} \cdot K_{S}\right\rangle\right) / 2} \gamma_{c_{1}, N, g}^{S}$. We denote $\Phi_{c_{1}}^{S, L}:=\sum_{N \geq 0} \Phi_{c_{1}, N}^{S, L}$. We denote by $p t \in H_{0}(S, \mathbb{Z})$ the class of a point. Sometimes we will consider the Donaldson invariants as polynomials on $H_{2}(S, \mathbb{Q})$ by putting $\Phi_{c_{1}, N, r}^{S, L}(\alpha):=\Phi_{c_{1}, N}^{S, L}\left(p t^{r} \alpha^{N-2 r}\right)$ for $\alpha \in H_{2}(S, \mathbb{Q})$.

If the moduli space $M_{L}\left(c_{1}, c_{2}\right)$ fulfills certain properties (in particular there is a universal sheaf $\mathcal{U}$ over $\left.S \times M_{H}\left(c_{1}, c_{2}\right)\right)$, then for $\alpha_{1}, \ldots \alpha_{r} \in H_{2 i}(S, \mathbb{Q})$ we have

$$
\Phi_{c_{1}, N}^{S, L}\left(\alpha_{1} \ldots \alpha_{r}\right)=\int_{M_{L}\left(c_{1}, c_{2}\right)} \nu\left(\alpha_{1}\right) \cdot \ldots \cdot \nu\left(\alpha_{r}\right)
$$

where $\nu(\alpha)=\left(c_{2}(\mathcal{U})-c_{1}^{2}(\mathcal{U}) / 4\right) / \alpha([\mathrm{Mo},[\mathrm{Li}])$.

We will use a result from $[\mathrm{E}-\mathrm{G}]$ (also proved independently in $[\mathrm{F}-\mathrm{Q}]$ ), We state it only for rational surfaces. Note that there are some changes in notation.

Definition 2.5. Let $\xi \in H^{2}(S, \mathbb{Z})$ be a class defining a good wall of type $\left(c_{1}, c_{2}\right)$. For $N:=$ $4 c_{2}-c_{1}^{2}-3$ we denote $d_{\xi, N}:=\left(N+3+\xi^{2}\right) / 4, e_{\xi, N}:=-\left\langle\xi \cdot\left(\xi-K_{S}\right)\right\rangle / 2+d_{\xi, N}+1$. Assume now that $\left(c_{1}, c_{2}\right)$ are fixed. Let

$$
T_{\xi}:=\operatorname{Hilb}^{d_{\xi, N}}(S \sqcup S)=\coprod_{n+m=d_{\xi, N}} \operatorname{Hilb}^{n}(S) \times \operatorname{Hilb}^{m}(S) .
$$

be the Hilbert scheme of $d$ points on 2 disjoint copies of $S$. Let $q: S \times T_{\xi} \longrightarrow T_{\xi}$ and $p: S \times T_{\xi} \longrightarrow T_{\xi}$ be the projections. Let $V_{\xi}$ be the sheaf $p^{*}\left(\mathcal{O}_{S}(-\xi) \oplus \mathcal{O}_{S}\left(-\xi+K_{S}\right)\right)$ on $S \times T_{\xi}$. Let $\mathcal{Z}_{1}$ (resp. $\left.\mathcal{Z}_{2}\right)$ be the subscheme of $S \times T_{\xi}$ which restricted to each component $S \times \operatorname{Hilb}^{n}(S) \times \operatorname{Hilb}^{m}(S)$ is the pullback of the universal subscheme $Z_{n}(S)$ (resp. $Z_{m}(S)$ ) from the first and second (resp. first and third) factor. Let $\mathcal{I}_{\mathcal{Z}_{1}}, \mathcal{I}_{\mathcal{Z}_{2}}$ be the corresponding ideal sheaves and $\left[\mathcal{Z}_{1}\right]$ and $\left[\mathcal{Z}_{2}\right]$ their cohomology classes. For $\alpha \in H_{i}(S, \mathbb{Q})$ let $\widetilde{\alpha}:=\left(\left[\mathcal{Z}_{1}\right]+\left[\mathcal{Z}_{2}\right]\right) / \alpha \in H^{4-i}\left(T_{\xi}, \mathbb{Q}\right)$ Then for $\alpha=\alpha_{1} \cdot \ldots \cdot \alpha_{N-2 r} p t^{r} \in A_{N}(S)$ (with $\alpha_{i} \in H_{2}(S, \mathbb{Q})$ ) we put

$$
\delta_{\xi, N}(\alpha):=\int_{T_{\xi}}\left(\left(\prod_{i=1}^{N-2 r}\left(\left\langle\alpha_{i}, \xi / 2\right\rangle+\widetilde{\alpha}_{i}\right)\right)(-1 / 4+\widetilde{p t})^{r} s\left(\operatorname{Ext}_{q}^{1}\left(\mathcal{I}_{\mathcal{Z}_{1}}, \mathcal{I}_{\mathcal{Z}_{2}} \otimes V_{\xi}\right)\right),\right.
$$

where $s(\cdot)$ denotes the total Segre class. We denote $\delta_{\xi}:=\sum_{N>0} \delta_{\xi, N}$. We will also denote for $\alpha \in H_{2}(S, \mathbb{Q})$ by $\delta_{\xi, N, r}(\alpha):=\delta_{\xi, N}\left(p t^{r} \alpha^{N-2 r}\right)$.

Theorem 2.6. [E-G], [E-Q] Let $S$ be a rational surface. Let $c_{1} \in H^{2}(S, \mathbb{Z})$ and $c_{2} \in \mathbb{Z}$. Let $H_{-}$and $H_{+}$be ample divisors on $S$, such that all the walls defined by elements of $W_{\left(c_{1}, c_{2}\right)}\left(H_{-}, H_{+}\right)$are good. Then for all $\alpha \in A_{N}(S)$ we have

$$
\Phi_{H_{+}, N}^{S}(\alpha)-\Phi_{H_{-}, N}^{S}(\alpha)=\sum_{\xi \in W_{\left(c_{1}, c_{2}\right)}\left(H_{-}, H_{+}\right)}(-1)^{e_{\xi, N}} \delta_{\xi}(\alpha) .
$$


2.3. Blowup formulas. We briefly recall the blowup formulas in the context of algebraic surfaces. In the case $b_{+}(S)>1$, when the invariants do not depend on the chamber structure, they have been shown e.g. in [0], [4] and in the most general form in $\left[\mathrm{F}-\mathrm{S}\right.$. In the case $b_{+}(S)=1$ we cite these results after [K-L]. By [T] the formulas of [F-S] also hold for $S$ with $b_{+}(S)=1$, if the chamber structure is properly taken into account. Let $S$ be an algebraic surface with $b_{+}=1$ and let $\epsilon: \widehat{S} \longrightarrow S$ be the blowup in a point. Let $E \in H^{2}(S, \mathbb{Z})$ be the class of the exceptional divisor. Let $c_{1} \in H^{2}(S, \mathbb{Z})$ and $c_{2} \in H^{4}(S, \mathbb{Z})$ and put $N=4 c_{2}-c_{1}^{2}-3$. Let $\mathcal{C} \subset \mathcal{C}_{\mathcal{S}}$ be a chamber of type $\left(c_{1}, c_{2}\right)$, let $\mathcal{C}_{\mathcal{E}} \subset \mathcal{C}_{\widehat{\mathcal{S}}}$ be a chamber of type $\left(c_{1}+E, c_{2}\right)$, and let $\mathcal{C}_{\boldsymbol{l}} \subset \mathcal{C}_{\widehat{\mathcal{S}}}$ be a chamber of type $\left(c_{1}, c_{2}\right)$. Following $[\mathbb{K}]$ we say that the chambers $\mathcal{C}$ and $\mathcal{C}_{\mathcal{E}}$ (resp. $\mathcal{C}$ and $\mathcal{C}_{l}$ ) are related chambers if $\epsilon^{*}(\mathcal{C})$ is contained in the closure $\overline{\mathcal{C}}_{E}$ (resp in $\overline{\mathcal{C}}_{0}$ ).

Theorem 2.7. There are universal polynomials $S_{k}(x)$ and $B_{k}(x)$ such that for all related chambers $\mathcal{C}$ and $C_{E}\left(\right.$ resp, $\mathcal{C}$ and $\left.\mathcal{C}_{1}\right)$ as above, all $k \leq N$ and all $\alpha \in A_{N-k}(S)$ we have

$$
\begin{aligned}
\Phi_{c_{1}-E}^{\widehat{S}, \mathcal{C}_{\mathcal{E}}}\left(\check{E}^{k} \alpha\right)=-\Phi_{c_{1}+E}^{\widehat{S}, \mathcal{C}_{\mathcal{E}}}\left(\check{E}^{k} \alpha\right) & =\Phi_{c_{1}}^{S, \mathcal{C}}\left(S_{k}(p t) \alpha\right), \\
\Phi_{c_{1}}^{\widehat{S}, \mathcal{C}_{1}}\left(\check{E}^{k} \alpha\right) & =\Phi_{c_{1}}^{S, \mathcal{C}}\left(B_{k}(p t) \alpha\right) .
\end{aligned}
$$

(Note the different sign convention). The $S_{k}(x)$ and $B_{k}(x)$ can be given in terms of the coefficients of of the q-development of certain $\sigma$-functions.

We refer to (2.7.1) as $S O(3)$-blowup formulas and to 2.7 .2 as $S U(2)$-blowup formulas. We will use that the $S_{k}(x)$ and the $B_{k}(x)$ are determined by recursive relations: (a1) $S_{2 k}(x)=0$ for all $k$, (b1) $S_{1}(x)=1, S_{3}(x)=-x, S_{5}(x)=x^{2}+2, S_{7}(x)=-x^{3}-6 x$, (a2) $B_{2 k+1}(x)=0$ for all $k,(\mathrm{~b} 2)$ $B_{0}(x)=1, B_{2}(x)=0, B_{4}(x)=-2$ and, in both cases, the recursive relation

$$
\begin{aligned}
& \sum_{i=0}^{h}\left(\begin{array}{c}
h \\
i
\end{array}\right)\left(U_{h+4-i} U_{i}-4 U_{h+3-i} U_{i+1}+6 U_{h+2-i} U_{i+2}-4 U_{h+1-i} U_{i+3}+U_{h-i} U_{i+4}\right) \\
& =-4 \sum_{i=0}^{h}\left(\begin{array}{c}
h \\
i
\end{array}\right)\left(x U_{h+2-i} U_{i}+x U_{h-i} U_{i+2}-2 x U_{h+1-i} U_{i+1}+U_{h-i} U_{i}\right),
\end{aligned}
$$

with either $U_{i}=S_{i}(x)$ or $U_{i}=B_{i}(x)$ (see e.g. [F-S, [K-L).

2.4. The walls for rational surfaces. Now let $S$ be a rational surface. We want to collect some information about the set of walls in the ample cone $C_{S}$. The following is easy to see:

Remark 2.8. 1. If $S$ is a rational ruled surface then $C_{S}=C_{S, g}$, i.e. all walls are good.

2. If $S$ is obtained from $\mathbb{P}_{2}$ by a sequence of blow ups with exceptional divisors $E_{1}, \ldots, E_{r}$ then $C_{S, g}=C_{S} \cap\left\{a\left(H-a_{1} E_{1}-\ldots-a_{r} E_{r}\right) \mid a>0, a_{i}>0, \sum_{i} a_{i}<3\right\}$.

Lemma 2.9. For any pair $\left(H_{-}, H_{+}\right)$of ample divisors on a rational surface $S$ and all $c_{1} \in \operatorname{Pic}(S)$ and $c_{2} \in H^{2}(S, \mathbb{Z})$ the set $W_{\left(c_{1}, c_{2}\right)}\left(H_{-}, H_{+}\right)$is finite.

Proof. The set $\left\{t H_{-}+(1-t) H_{+} \mid t \in[0,1]\right\}$ is a compact subset of $C_{S}$. Therefore by [F-M corollary 1.6 it intersects only finitely many walls of type $\left(c_{1}, c_{2}\right)$. 
We now give a list of all walls for $S=\widehat{\mathbb{P}}_{2}$ and $S=\mathbb{P}_{1} \times \mathbb{P}_{1}$ which will be used repeatedly in our computations. We denote by $F=H-E$ the class of a fibre of $\widehat{\mathbb{P}}_{2} \longrightarrow \mathbb{P}_{1}$. We also denote by $F$ the fibre of the projection to the first factor of $\mathbb{P}_{1} \times \mathbb{P}_{1}$ and by $G$ the class of the fibre of the projection to the second factor. The verifications are elementary.

\section{Remark 2.10.}

$$
\begin{aligned}
W_{0, c_{2}}^{\widehat{\mathbb{P}}_{2}}(F, H-\delta E) & =\left\{2 a H-2 b E \mid b>a>\delta b, b^{2}-a^{2} \leq c_{2}\right\}, \\
W_{E, c_{2}}^{\widehat{\mathbb{P}}_{2}}(F, H-\delta E) & =\left\{2 a H-(2 b-1) E \mid b>a>\delta(b-1 / 2), b(b-1)-a^{2} \leq c_{2}\right\}, \\
W_{H, c_{2}}^{\widehat{\mathbb{P}}_{2}}(F, H-\delta E) & =\left\{(2 a-1) H-2 b E \mid b \geq a>\delta b+1 / 2, b^{2}-a(a-1) \leq c_{2}\right\}, \\
W_{F, c_{2}}^{\widehat{\mathbb{P}}_{2}}(F, H-\delta E) & =\left\{(2 a-1) H-(2 b-1) E \mid b>a>\delta(b-1 / 2)+1 / 2, b(b-1)-a(a-1) \leq c_{2}\right\}, \\
W_{0, c_{2}}^{\mathbb{P}_{1} \times \mathbb{P}_{1}}(F, F+\delta G) & =\left\{2 a F-2 b G \mid 0<b<a \delta, 2 a b \leq c_{2}\right\}, \\
W_{F, c_{2}}^{\mathbb{P}_{1} \times \mathbb{P}_{1}}(F, F+\delta G) & =\left\{(2 a-1) F-2 b G \mid 0<b<(a-1 / 2) \delta,(2 a-1) b \leq c_{2}\right\}, \\
W_{G, c_{2}}^{\mathbb{P}_{1} \times \mathbb{P}_{1}}(F, F+\delta G) & =\left\{2 a F-(2 b-1) G \mid 0<b<a \delta+1 / 2,(2 b-1) a \leq c_{2}\right\}, \\
W_{F+G, c_{2}}^{\mathbb{P}_{1} \times \mathbb{P}_{1}}(F, F+\delta G) & =\left\{(2 a-1) F-(2 b-1) G \mid 0<b<(a-1 / 2) \delta+1 / 2,2 a b-a-b \leq c_{2}\right\} .
\end{aligned}
$$

2.5. Botts formula. Now we recall the Bott residue formula (see e.g. [B], A-B], [E-S2], C-L1], C-L2]). Let $X$ be a smooth projective variety of dimension $n$ with an algebraic action of the multiplicative group $\mathbb{C}^{*}$ such that the fixpoint set $F$ is finite. Differentiation of the action induces a global vector field $\xi \in H^{0}\left(X, T_{X}\right)$, and $F$ is precisely the zero locus of $\xi$. Hence the Koszul complex on the map $\xi^{\vee}: \Omega_{X} \longrightarrow \mathcal{O}_{X}$ is a locally free resolution of $\mathcal{O}_{F}$. For $i \geq 0$ denote by $B_{i}$ the cokernel of the Koszul map $\Omega_{X}^{i+1} \longrightarrow \Omega_{X}^{i}$. It is well known that $H^{j}\left(X, \Omega_{X}^{i}\right)=0$ for $i \neq j$. So there are natural exact sequences for all $i$ :

$$
0 \longrightarrow H^{i}\left(X, \Omega_{X}^{i}\right) \stackrel{p_{i}}{\longrightarrow} H^{i}\left(X, B_{i}\right) \stackrel{r_{i}}{\longrightarrow} H^{i+1}\left(X, B_{i+1}\right) \longrightarrow 0 .
$$

In particular there are natural maps $q_{i}=r_{i-1} \circ \ldots \circ r_{0}: H^{0}\left(F, \mathcal{O}_{F}\right) \longrightarrow H^{i}\left(X, B_{i}\right)$.

Definition 2.11. Let $f: F \longrightarrow \mathbb{C}$ be a function and $c \in H^{i}\left(X, \Omega_{X}^{i}\right)$. We say that $f$ represents $c$ if $q_{i+1}(f)=0$ and $q_{i}(f)=p_{i}(c)$.

If $f_{1}$ represents $a_{1} \in H^{i}\left(X, \Omega_{X}^{i}\right)$ and $f_{2}$ represents $a_{2} \in H^{j}\left(X, \Omega_{X}^{j}\right)$, then $f_{1} f_{2}$ represents $a_{1} \cdot a_{2} \in$ $H^{i+j}\left(X, \Omega_{X}^{i+j}\right)$.

The following result enables us to compute the degree of polynomials of weight in the Chern classes of equivariant vector bundles on $X$. Let $\mathcal{E}$ be an equivariant vector bundle of rank $r$ on $X$. At each fixpoint $x \in F$ the fibre $\mathcal{E}(x)$ splits as a direct sum of one-dimensional representations of $\mathbb{C}^{*}$. Let $\tau_{1}(E, x), \ldots \tau_{r}(\mathcal{E}, x)$ denote the corresponding weights, and for all $k \geq 0$ let $\sigma_{k}(\mathcal{E}, x) \in \mathbb{Z}$ be the $k$-th elementary symmetric function in the $\tau_{i}(\mathcal{E}, x)$.

Theorem 2.12. $\quad$ 1. The $k$-th Chern class $c_{k}(\mathcal{E}) \in H^{k}\left(X, \Omega_{X}^{k}\right)$ of $\mathcal{E}$ can be represented by the function $x \mapsto \sigma_{k}(\mathcal{E}, x)$.

2. The composition $H^{0}\left(\mathcal{O}_{F}\right) \longrightarrow H^{n}\left(X, \Omega_{X}^{n}\right) \stackrel{\text { res }}{\longrightarrow} \mathbb{C}$ maps $f: F \longrightarrow \mathbb{C}$ to $\sum_{x \in F} f(x) / \sigma_{n}\left(T_{X}, x\right)$. 


\section{Applichtion of the Bott Residue formula}

In this section we want to see how the Bott residue formula can be used to compute $\delta_{\xi, N}$ for a class $\xi$ defining a wall on a rational surface $X$. Let $\Gamma=\mathbb{C}^{*} \times \mathbb{C}^{*}$ be an algebraic 2-torus and let $\lambda$ and $\mu$ be two independent primitive characters of $\Gamma$. We identify the representation ring of $\Gamma$ with the ring of Laurent polynomials in $\lambda$ and $\mu$. For a variety $Y$ with an action of $\Gamma$ we will denote by $F_{Y}$ the set of fixpoints.

3.1. Actions of a torus on rational surfaces. We are going to define actions with finitely many fixpoints of $\Gamma$ on $X=\mathbb{P}_{2}, X=\mathbb{P}_{1} \times \mathbb{P}_{1}$ and inductively on surfaces $X=X_{r}$, where $X_{0}=\mathbb{P}_{2}$ or $X_{0}=\mathbb{P}_{1} \times \mathbb{P}_{1}$ and $X_{i}$ is the blowup of a fixpoint of the $\Gamma$-action on $X_{i-1}$. We also define a lift of the action of $\Gamma$ to all line bundles on $X$. These actions will have the following properties:

1. Each fixpoint $p \in F_{X}$ has an invariant neighbourhood $A_{p}$ isomorphic to $\mathbb{A}^{2}=\operatorname{spec}([k[x, y])$ on which $\Gamma$ acts by $t \cdot x=\alpha_{p} x, t \cdot y=\beta_{p} y$ for two independent characters $\alpha_{p}$ and $\beta_{p}$ of $\Gamma$, and the $A_{p}$ cover $X$.

2. For each line bundle $L \in \operatorname{Pic}(X)$ the restriction $\left.L\right|_{A_{p}}$ has a nowhere vanishing section $s_{L, p}$, with $t \cdot s_{L, p}=\gamma_{L, p} s_{L, p}$ for $\gamma_{L, p}$ a character of $\Gamma$.

(a) The case of $\mathbb{P}_{2}$. Let $T_{0}, T_{1}, T_{2}$ be homogeneous coordinates on $\mathbb{P}_{2}$. Let $\Gamma$ act on $\mathbb{P}_{2}$ by $t \cdot T_{0}=T_{0}$, $t \cdot T_{1}=\lambda T_{1}, t \cdot T_{2}=\mu T_{2}$. The action of $\Gamma$ has 3 fixpoints $p_{0}:=(1: 0: 0), p_{1}:=(0: 1: 0)$ and $p_{2}:=(0: 0: 1)$. The sets $A_{p_{i}}:=D\left(T_{i}\right)$ (i.e. the locus where $T_{i} \neq 0$ ) are affine invariant neighbourhoods. In appropriate coordinates $x, y$ on $A_{p_{0}}$ (resp. $\left.A_{p_{1}}, A_{p_{2}}\right)$, the induced action of $\Gamma$ is $t \cdot(x, y)=(\lambda x, \mu y)$ (resp. $\left.t \cdot(x, y)=\left(\lambda^{-1} x, \mu \lambda^{-1} y\right), t \cdot(x, y)=\left(\mu^{-1} x, \lambda \mu^{-1} y\right)\right)$. Furthermore on $A_{p_{i}}$ the monomial $T_{i}^{n}$ defines a trivializing section of $\mathcal{O}_{\mathbb{P}_{2}}(n)$ with $t \cdot T_{0}^{n}=T_{0}^{n}, t \cdot T_{1}^{n}=\lambda^{n} T_{1}^{n}, t \cdot T_{2}^{n}=\mu^{n} T_{2}^{n}$.

(b) The case of $\mathbb{P}_{1} \times \mathbb{P}_{1}$. Let $X_{0}, X_{1}$ and $Y_{0}, Y_{1}$ be homogeneous coordinates on the two factors. Let $\Gamma$ act on $\mathbb{P}_{1} \times \mathbb{P}_{1}$ by $t \cdot X_{0}=X_{0}, t \cdot X_{1}=\lambda X_{1}, t \cdot Y_{0}=Y_{0}$ and $t \cdot Y_{1}=\mu Y_{1}$. This action has 4 fixpoints $p_{i j}:=V\left(X_{1-i}\right) \cap V\left(Y_{1-j}\right)$ (i.e. the locus where $X_{1-i}=Y_{1-j}=0$ ), which have affine neightbourhoods $A_{p_{i j}}=D\left(X_{i}\right) \cap D\left(Y_{j}\right)$. In the appropriate coordinates $x, y$ on $A_{p_{i j}}$ the action is given by $t \cdot(x, y)=\left(\lambda^{1-2 i} x, \mu^{1-2 j} y\right)(i$ and $j \in\{0,1\})$. Finally a trivializing section of $\mathcal{O}(n, m)$ on $A_{p_{i j}}$ is $X_{i}^{n} Y_{i}^{m}$ with $t \cdot\left(X_{i}^{n} Y_{j}^{m}\right)=\lambda^{i n} \mu^{j m} X_{i}^{n} Y_{j}^{m}$.

(c) The blowup. Now assume that $Y$ is a surface obtained from $\mathbb{P}_{1} \times \mathbb{P}_{1}$ or $\mathbb{P}_{2}$ by successively blowing up fixpoints of the action of $\Gamma$, and assume that the action is extended to $Y$, so that it still has finitely many fixpoints, and that the assumptions (1) and (2) above are satisfied. Let $p \in F_{Y}$ be a fixpoint. Let $A_{p}$ be an affine neighbourhood of $p$ with coordinates $x, y$ on which $\Gamma$ acts by $t \cdot(x, y)=(\alpha x, \beta y)$ for two independent characters $\alpha, \beta$ of $\Gamma$. Let $X$ be the blowup of $Y$ in $p$, and denote by $E$ the exceptional divisor and by $\widehat{A}$ the blow up of $A_{p}$ at $p$. We can identify $E=\mathbb{P}\left(\langle x, y\rangle^{\vee}\right)$, and the induced action of $\Gamma$ on $E$ has 2 fixpoints $q_{0}:=(1: 0)$ and $q_{1}:=(0: 1)$, which are the fixpoints of $\Gamma$ on $X$ over $p$. There are affine neighbourhoods $A_{q_{0}}=\widehat{A} \cap D(x)$ and $A_{q_{1}}=\widehat{A} \cap D(y)$ of $q_{0}$ and $q_{1}$ in $X$, with coordinates $(x, y / x)$ and $(y, x / y)$. The action $t \cdot(x, y / x)=\left(\alpha x, \beta \alpha^{-1} y / x\right)$, $t \cdot(y, x / y)=\left(\beta y, \alpha \beta^{-1} x / y\right)$ extends the action of $\Gamma$ on $Y \backslash\{p\}$ to $X$. Let $L$ be a linebundle on $Y$ with a trivializing section $s_{L, q}$ near each $q \in F_{Y}$ with $t \cdot s_{L, q}=\gamma_{L, p_{i}} s_{L, q}$. Then $L \otimes \mathcal{O}(k E)$ has for 
$i \neq 0$ still $s_{L, q}$ as a trivializing section near $q$ (with $q \neq p$ ), and near $q_{0}$ (resp. $q_{1}$ ) such a section is $s_{0}=s_{L, p} \otimes y^{-k}\left(\right.$ resp. $\left.s_{1}=s_{L, p} \otimes x^{-k}\right)$ with $t \cdot s_{0}=\gamma_{L, p} \beta^{-k} s_{0}\left(\right.$ resp. $\left.t \cdot s_{1}=\gamma_{L, p} \alpha^{-k} s_{1}\right)$.

3.2. The induced action on the Hilbert scheme. We assume that $S$ is a surface obtained by blowing up $\mathbb{P}_{2}$ or $\mathbb{P}_{1} \times \mathbb{P}_{1}$ repeatedly, with an action of $\Gamma$ as above. We fix a positive integer $d$ and want to study the induced action of $\Gamma$ on the Hilbert scheme $\operatorname{Hilb}^{d}(S \sqcup S)$ and on certain "standard bundles" on $\operatorname{Hilb}^{d}(S \sqcup S)$, which appear in the wall-crossing formula 2.5. The induced action on $\operatorname{Hilb}^{d}(S \sqcup S)$ is given by $t \cdot(Y, Z)=(t \cdot Y, t \cdot Z)$, where for a subscheme $Z \subset S$ we denote by $t \cdot Z$ the subscheme with ideal $t \cdot \mathcal{I}_{Z / S}:=\left\{t \cdot f \mid f \in \mathcal{I}_{Z / S}\right\}$.

Now let $F_{S}:=\left\{p_{1}, \ldots, p_{m}\right\}$ be the set of fixpoints on $S$, and, for all $i$, let $A_{i}$ be the invariant affine neighbourhood of $p_{i}$ with coordinates $x_{i}, y_{i}$, such that $t \cdot x_{i}=\alpha_{i} x_{i}, t \cdot y_{i}=\beta_{i} y_{i}$ for two independent characters $\alpha_{i}$ and $\beta_{i}$. As the characters $\alpha_{i}$ and $\beta_{i}$ are independent, it is easy to see that a subscheme $Z \in \operatorname{Hilb}^{n}(S)$ is fixed by the induced action of $\Gamma$ if and only if $\operatorname{supp}(Z) \subset F_{S}$ and if, for all $i$, denoting by $Z_{i}$ the part of $Z$ with support $p_{i}$, all the ideals $\mathcal{I}_{Z_{i} / A_{i}}$ are generated by monomials in $x_{i}$ and $y_{i}$. We denote by $F_{\mathrm{Hilb}^{d}(S \sqcup S)}$ the fixpoints on $\operatorname{Hilb}^{d}(S \sqcup S)$.

Definition 3.1. A partition of a nonnegative integer $n$ is a sequence $\alpha=\left(a_{0}, \ldots a_{r}\right)$ with $\alpha_{0} \geq$ $\ldots \geq a_{r-1} \geq a_{r}=0$ and $\sum a_{i}=n$. We identify $\left(a_{0}, \ldots a_{r}\right)$ and $\left(a_{0}, \ldots a_{r}, 0\right)$. Let $P_{2 m}(d)$ be the set of sequences $\left(P_{1}, \ldots, P_{m}, Q_{1}, \ldots Q_{m}\right)$ where the $P_{i}$ and $Q_{i}$ are all partitions of numbers $n_{i}$ and $m_{i}$ with $\sum\left(n_{i}+m_{i}\right)=d$. We see that $P_{2 m}(d)$ and $F_{\mathrm{Hilb}^{d}(S \sqcup S)}$ are in one-one correspondence, with $\left(P_{1}, \ldots, P_{m}, Q_{1}, \ldots Q_{m}\right)$ corresponding to $\left(Y_{1} \sqcup \ldots \sqcup Y_{m}, Z_{1} \sqcup \ldots \sqcup Z_{m}\right)$, where for $P_{i}=\left(a_{0}, \ldots, a_{r}\right), Q_{i}=\left(b_{0}, \ldots, b_{r}\right)$ the subschemes $Y_{i}$ and $Z_{i}$ are supported at $p_{i}$ and defined by $\mathcal{I}_{Y_{i} / A_{i}}=\left(y_{i}^{a_{0}}, x_{i} y_{i}^{a_{1}}, \ldots x_{i}^{s} y_{i}^{a_{s}}, x^{s+1}\right)$ and $\mathcal{I}_{Z_{i} / A_{i}}=\left(y_{i}^{b_{0}}, x_{i} y_{i}^{b_{1}}, \ldots x_{i}^{r} y_{i}^{b_{r}}, x^{r+1}\right)$.

3.3. The action on some standard bundles. We now want to determine the action of $\Gamma$ on some standard bundles on $\operatorname{Hilb}^{d}(S \sqcup S)$ which appear in the wall-crossing formula 2.5. Let $\xi$ define a good wall. We denote by $V$ the vector bundle $\mathcal{O}_{S}(-\xi) \oplus \mathcal{O}_{S}\left(-\xi+K_{S}\right)$. Then by the results of E-G] and [F-Q] $\operatorname{Ext}_{q}^{1}\left(\mathcal{I}_{\mathcal{Z}_{1}}, \mathcal{I}_{\mathcal{Z}_{2}} \otimes p^{*} V\right)$ is a locally free sheaf on $\operatorname{Hilb}^{d}(S \sqcup S)$, which is compatibel with base change, i.e. its fibre over $(Y, Z) \in \operatorname{Hilb}^{d}(S \sqcup S)$ is $\operatorname{Ext}^{1}\left(\mathcal{I}_{Y}, \mathcal{I}_{Z} \otimes V\right)$. Furthermore the $\Gamma$-linearisation of $\mathcal{O}_{S}(\xi)$ from 3.1 determines in a canonical way a $\Gamma$-linearisation of $\operatorname{Ext}_{q}^{1}\left(\mathcal{I}_{\mathcal{Z}_{1}}, \mathcal{I}_{\mathcal{Z}_{2}} \otimes p^{*} V\right)$. It also induces an action of $\Gamma$ on $H^{1}(S, V)$. Now let $(Y, Z) \in F_{\mathrm{Hilb}^{d}(S \sqcup S)}$ be a point corresponding to $\left(P_{1}, \ldots, P_{m}, Q_{1}, \ldots Q_{m}\right)$. We will determine the action on the fibre $\operatorname{Ext}^{1}\left(\mathcal{I}_{Y}, \mathcal{I}_{Z} \otimes V\right)$. We denote by $V\left(p_{i}\right)$ the fibre of $V$ over the fixpoint $p_{i}$ considered as a representation of $\Gamma$.

Lemma 3.2. For partitions $P:=\left(a_{0}, \ldots, a_{r}\right), Q:=\left(b_{0}, \ldots, b_{r}\right)$ we denote

$$
E_{P, Q}(x, y):=\sum_{1 \leq i \leq j \leq r}\left(\sum_{s=a_{j}}^{a_{j-1}-1} x^{i-j-1} y^{b_{i-1}-s-1}+\sum_{s=b_{j}}^{b_{j-1}-1} x^{j-i} y^{s-a_{i-1}}\right) .
$$


Then in the representation ring of $\Gamma$ we have the identities

$$
\begin{aligned}
T_{H i l b^{d}(S \sqcup S)}(Y, Z) & =\sum_{i=0}^{m}\left(E_{P_{i}, P_{i}}\left(\alpha_{i}, \beta_{i}\right)+E_{Q_{i}, Q_{i}}\left(\alpha_{i}, \beta_{i}\right)\right), \\
\operatorname{Ext}^{1}\left(\mathcal{I}_{Y}, \mathcal{I}_{Z} \otimes V\right) & \left.=H^{1}(S, V)+\sum_{i=0}^{m} V\left(p_{i}\right) \cdot E_{P_{i}, Q_{i}}\left(\alpha_{i}, \beta_{i}\right)\right) .
\end{aligned}
$$

Proof. 3.2.1 follows directly from E-S1.

Claim: In the representation ring of $\Gamma$ we have the identity

$\operatorname{Ext}^{1}\left(\mathcal{I}_{Y}, \mathcal{I}_{Z} \otimes V\right)=H^{1}(S, V)+H^{0}\left(S, \mathcal{E} \S \sqcup^{1}\left(\mathcal{I}_{Y}, \mathcal{I}_{Z}\right) \otimes V\right)+H^{0}\left(S, \mathcal{O}_{Z} \otimes V\right)-H^{0}\left(S, \mathcal{H} 2 \Uparrow\left(\mathcal{O}_{Y}, \mathcal{O}_{Z}\right) \otimes V\right)$.

Proof of the Claim: As $\xi$ defines a good wall, we have $H^{2}\left(S, \mathcal{H} 2 \mathfrak{\llbracket}\left(\mathcal{I}_{Y}, \mathcal{I}_{Z}\right) \otimes V\right)=H^{0}\left(S, \mathcal{H} 2 \Uparrow\left(\mathcal{I}_{Z}, \mathcal{I}_{Y}\right) \otimes\right.$ $\left.V^{\vee}\left(K_{S}\right)\right)=0$ and $H^{0}\left(S, \mathcal{H} 2 \mathfrak{I}\left(\mathcal{I}_{Z}, \mathcal{I}_{Y}\right) \otimes V\right)=0$. Therefore the low-term exact sequence of the local to global spectral sequence $H^{p}\left(\mathcal{E} \S \sqcup^{q}\left(\mathcal{I}_{Y}, \mathcal{I}_{Z} \otimes V\right)\right) \Rightarrow \operatorname{Ext}^{p+q}\left(\mathcal{I}_{Y}, \mathcal{I}_{Z} \otimes V\right)$ gives in the representation ring of $\Gamma$

$$
\operatorname{Ext}^{1}\left(\mathcal{I}_{Y}, \mathcal{I}_{Z}(V)\right)=H^{0}\left(S, \mathcal{E} \S \sqcup^{1}\left(\mathcal{I}_{Y}, \mathcal{I}_{Z}\right) \otimes V\right)+H^{1}\left(S, \mathcal{H}\left(\Uparrow\left(\mathcal{I}_{Y}, \mathcal{I}_{Z}\right) \otimes V\right) .\right.
$$

We have an exact sequence

$$
0 \longrightarrow \mathcal{I}_{Z} \longrightarrow \mathcal{H} 2 \Uparrow\left(\mathcal{I}_{Y}, \mathcal{I}_{Z}\right) \longrightarrow \mathcal{H} 2 \mathbb{\llbracket}\left(\mathcal{O}_{Y}, \mathcal{O}_{Z}\right) \longrightarrow 0
$$

So, tensoring by $V$, taking the long exact sequence of cohomology and using the vanishing of $H^{0}\left(\mathcal{H} 2 \mathfrak{\Sigma}\left(\mathcal{I}_{Y}, \mathcal{I}_{Z}\right) \otimes V\right)$ and $H^{1}\left(\mathcal{H}\left(\mathfrak{I}\left(\mathcal{O}_{Y}, \mathcal{O}_{Z}\right) \otimes V\right)\right.$, we get in the representation ring of $\Gamma$ the identity

$$
H^{1}\left(\mathcal{H} 2 \uparrow\left(\mathcal{I}_{Y}, \mathcal{I}_{Z}\right) \otimes V\right)=H^{1}\left(S, \mathcal{I}_{Z} \otimes V\right)-H^{0}\left(\mathcal{H} 2 \Uparrow\left(\mathcal{O}_{Y}, \mathcal{O}_{Z}\right) \otimes V\right) .
$$

Finally we use the sequence $0 \longrightarrow \mathcal{I}_{Z} \otimes V \longrightarrow V \longrightarrow \mathcal{O}_{Z} \otimes V \longrightarrow 0$ and the vanishing of $H^{0}(S, V)$ and $H^{1}\left(S, \mathcal{O}_{Z} \otimes V\right)$ to replace $H^{1}\left(S, \mathcal{I}_{Z} \otimes V\right)$ by $H^{0}\left(S, \mathcal{O}_{Z} \otimes V\right)+H^{1}(S, V)$. This shows the claim.

We denote by $\mathcal{F}$ the virtual $\Gamma$-sheaf $\mathcal{E} \S \sqcup^{1}\left(\mathcal{I}_{Y}, \mathcal{I}_{Z}\right)+\mathcal{O}_{Z}-\mathcal{H} 2 \mathfrak{\Perp}\left(\mathcal{O}_{Y}, \mathcal{O}_{Z}\right)$. We have to show that $H^{0}(S, \mathcal{F} \otimes V)=\sum_{i=0}^{m} V\left(p_{i}\right) \cdot E_{P_{i}, Q_{i}}\left(\alpha_{i}, \beta_{i}\right)$. If we denote by $\mathcal{F}_{i}$ the part of $\mathcal{F}$ with support $p_{i}$, then $H^{0}(S, \mathcal{F} \otimes V)=\sum_{i=1}^{m} H^{0}\left(S, \mathcal{F}_{i} \otimes V\right)$. We can therefore assume that $\operatorname{supp}(Y)=\operatorname{supp}(Z)$ is one fixpoint $p$. Let $x$ and $y$ be coordinates near $p$ as before and $R:=\mathbb{C}[x, y]$. Let $J:=\left(y^{a_{0}}, x y^{a_{1}}, \ldots, x^{r+1}\right)$ (resp. $\left.I:=\left(y^{b_{0}}, x y^{b_{1}}, \ldots, x^{r+1}\right)\right)$ be the ideal of $Y$ (resp. $Z$ ). We denote by $F$ the virtual $R$ - $\Gamma$ module corresponding to $\mathcal{F}$. In the representation ring of $\Gamma$ we have

$$
H^{0}(S, \mathcal{F} \otimes V)=F \cdot V(p) .
$$

So we finally have to show that in the representation ring of $\Gamma$ we have $F=E_{\left(a_{0}, \ldots, a_{r}\right),\left(b_{0}, \ldots b_{r}\right)}(\lambda, \mu)$. The exact sequences

$$
\begin{aligned}
& 0 \longrightarrow I \longrightarrow \operatorname{Hom}_{R}(J, I) \longrightarrow \mathcal{H} 2 \mathbb{\Perp}(R / J, R / I) \longrightarrow 0 \\
& 0 \longrightarrow I \longrightarrow R \longrightarrow R / I \longrightarrow 0
\end{aligned}
$$

give $F=\operatorname{Ext}_{R}^{1}(J, I)-\operatorname{Hom}_{R}(J, I)+R$ in the representation ring of $\Gamma$.

Following [E-S1] we denote by $R[\alpha, \beta]$ the ring $R$ with $\Gamma$-operation defined by $t\left(x^{i} y^{j}\right):=x^{i-\alpha} y^{j-\beta}$. We put $A_{0}:=\bigoplus_{i=0}^{r} R\left[i, a_{i}\right], B_{0}:=\bigoplus_{j=0}^{r} R\left[j, b_{j}\right], A_{1}:=\bigoplus_{i=1}^{r} R\left[i, a_{i-1}\right], B_{1}:=\bigoplus_{j=1}^{r} R\left[j, a_{j-1}\right]$. 
Then we have $\Gamma$-equivariant free resolutions $0 \longrightarrow A_{1} \longrightarrow A_{0} \longrightarrow J \longrightarrow 0$ and $0 \longrightarrow B_{1} \longrightarrow B_{0} \longrightarrow$ $I \longrightarrow 0$. So the total complex

$$
A_{0}^{\vee} \otimes B_{1} \stackrel{\alpha}{\longrightarrow} A_{1}^{\vee} \otimes B_{1} \oplus A_{0}^{\vee} \otimes B_{0} \longrightarrow A_{1}^{\vee} \otimes B_{0}
$$

associated to the double complex $\operatorname{Hom}_{R}\left(\mathcal{A}_{\bullet}, B_{\bullet}\right)$ computes the $\operatorname{Ext}_{R}^{i}(J, I)$, hence $F=R+A_{1}^{\vee} \otimes$ $B_{1}+A_{0}^{\vee} \otimes B_{0}-A_{0}^{\vee} \otimes B_{1}-A_{1}^{\vee} \otimes B_{0}$.

Again following [E-S1] we write $n_{i}:=\left(i, a_{i-1}\right), d_{i}:=\left(i, a_{i}\right), m_{j}:=\left(j, b_{j-1}\right)$ and $e_{j}:=\left(j, b_{j}\right)$. Then a calculation analogous to [E-S1] shows

$$
F=R+\sum_{\substack{1 \leq i \leq r \\ 0 \leq j \leq r}} R\left[e_{j}-n_{i}\right]-\sum_{\substack{1 \leq i \leq r \\ 1 \leq j \leq r}} R\left[m_{j}-n_{i}\right]-\sum_{\substack{0 \leq i \leq r \\ 0 \leq j \leq r}} R\left[e_{j}-d_{i}\right]+\sum_{\substack{0 \leq i \leq r \\ 1 \leq j \leq r}} R\left[m_{j}-d_{i}\right] .
$$

Putting

$$
\begin{aligned}
K_{i, j} & :=R\left[m_{j}-d_{i-1}\right]-R\left[m_{j}-n_{i}\right]-R\left[e_{j}-d_{i-1}\right]+R\left[e_{j}-n_{i}\right], \\
L_{i, j} & :=R\left[m_{i}-d_{j}\right]-R\left[m_{i}-n_{j}\right]-R\left[e_{i-1}-d_{j}\right]+R\left[e_{i-1}-n_{j}\right],
\end{aligned}
$$

a calculation analogous to E-S1 gives

$$
F=\sum_{1 \leq i \leq j \leq r}\left(K_{i, j}+L_{i, j}\right), K_{i, j}=\sum_{s=a_{i}}^{a_{i-1}} \lambda^{i-j-1} \mu^{b_{i-1}-s-1} \text { and } L_{i, j}=\sum_{s=b_{j}}^{b_{j-1}} \lambda^{j-i} \mu^{s-a_{i-1}},
$$

and the result follows.

We want to use the easy fact that representation of cohomology classes is compatible with equivariant pullback: Let $X$ and $Y$ be smooth projective varieties with an action of $\mathbb{C}^{*}$ with finitely many fixpoints and let $\mu: X \longrightarrow Y$ be an equivariant surjective morphism. Then $\mu$ induces a morphism $\left.\mu\right|_{F_{X}}: F_{X} \longrightarrow F_{Y}$.

Lemma 3.3. $f \in \mathcal{O}_{F_{Y}}$ represents a cohomology class $c \in H^{j}\left(Y, \Omega_{Y}^{j}\right)$ if and only if $\left(\left.\mu\right|_{F_{X}}\right)^{*} f$ represents $\mu^{*} c$.

Lemma 3.4. Let $\alpha \in H^{k}(S, \mathbb{Z})$ be a class represented by $f: F_{S} \longrightarrow \mathbb{C}$. Then $\widetilde{\alpha}$ (see 2.5) on $\operatorname{Hilb}^{d}(S \sqcup S)$ is represented by

$$
\tilde{f}: P_{2 m}(d) \longrightarrow \mathbb{C},\left(\left(P_{i}\right),\left(Q_{i}\right)\right) \mapsto \prod_{i=1}^{m}\left(n_{i}+m_{i}\right) f\left(p_{i}\right),
$$

where $P_{i} \in P\left(n_{i}\right)$ and $Q_{i} \in P\left(m_{i}\right)$.

Proof. Let

$$
\operatorname{Hilb}^{d-1, d}(S \sqcup S):=\left\{\left(Z_{d-1}, Z_{d}\right) \in \operatorname{Hilb}^{d-1}(S \sqcup S) \times \operatorname{Hilb}^{d}(S \sqcup S) \mid Z_{d-1} \subset Z_{d}\right\}
$$

with the reduced induced structure. Then $\operatorname{Hilb}^{d-1, d}(S \sqcup S)$ is smooth and we have a diagram

$$
\operatorname{Hilb}^{d}(S \sqcup S) \stackrel{\varphi}{\longleftarrow} \operatorname{Hilb}^{d-1, d}(S \sqcup S) \stackrel{\psi}{\longrightarrow}(S \sqcup S) \times \operatorname{Hilb}^{d-1}(S \sqcup S) \stackrel{\eta}{\longrightarrow} S \times \operatorname{Hilb}^{d-1}(S \sqcup S)
$$

Here $\psi$ is the blowup along the universal family $Z_{d}(S \sqcup S)$ 国 and $\eta$ is induced by the identity map on $S$ and $\operatorname{Hilb}^{d}(S \sqcup S)$. It is easy to see from the definitions that $\varphi^{*} \widetilde{\alpha}=\psi^{*} \eta^{*}\left(p_{1}^{*} \alpha+p_{2}^{*} \bar{\alpha}\right)$, where $p_{1}$ 
and $p_{2}$ are the projections of $S \times \operatorname{Hilb}^{d-1}(S \sqcup S)$ onto its two factors and $\bar{\alpha}$ is the class corresponding to $\widetilde{\alpha}$ if we replace $d$ by $d-1$. It is easy to see that $\varphi, \psi$ and $\eta$ are equivariant for the natural lifts of the action of $\Gamma$ on $S$, furthermore the fixpoint sets $F_{\mathrm{Hilb}^{d}(S \sqcup S)}, F_{\mathrm{Hilb}^{d-1, d}(S \sqcup S)}$ and $F_{S \times \operatorname{Hilb}^{d-1}(S \sqcup S)}$ are all finite. In fact we can identify

$$
F_{\mathrm{Hilb}^{d-1, d}(S \sqcup S)}=\left\{\left(\left(S_{i}, T_{i}\right),\left(P_{i}, Q_{i}\right)\right) \in P_{2 m}(d-1) \times P_{2 m}(d) \mid P_{i} \geq S_{i}, Q_{i} \geq T_{i} \text { for all } i\right\},
$$

where for partitions $P=\left(a_{1}, \ldots, a_{r}\right), Q=\left(b_{1}, \ldots b_{r}\right)$ we denote by $P \geq Q$ that $a_{i} \geq b_{i}$ for all $i$. Obviously $F_{S \times \mathrm{Hilb}^{d-1}(S \sqcup S)}=F_{S} \times P_{2 m}(d-1)$ and with this identification $\varphi$ and $\eta \circ \psi$ are the obvious maps. Now, applying lemma 3.3 to $\varphi$ and $\eta \circ \psi$, the result follows by easy induction.

We can now put our results together:

Notation 3.5. Fix a one-parameter subgroup $T$ of $\Gamma$. Let $\xi$ define a good wall on $S$. For any line bundle $L$ on $S$ denote by $w_{i}(L)$ the weight of the induced action of $T$ on the fibre $L\left(p_{i}\right)$. Let $L_{1}$ and $L_{2}$ be two line bundles with $\left\langle L_{1} \cdot L_{2}\right\rangle=p t$ (e.g. if $S$ is a blow up of $\mathbb{P}_{2}$ then we take $L_{1}=L_{2}=H$ ). Furthermore denote by $w\left(x_{i}\right), w\left(y_{i}\right)$ the weight of the action of $T$ on $x_{i}, y_{i}$. We denote for partitions $P=\left(a_{0}, \ldots a_{r}\right)$ and $Q=\left(b_{0}, \ldots b_{r}\right)$ of numbers $n$ and $m$

$$
\begin{gathered}
\bar{F}_{P, Q}(u, v):=\prod_{1 \leq i \leq j \leq r} \prod_{s=a_{j}}^{a_{j-1}-1}\left((i-j-1) u+\left(b_{i-1}-s-1\right) v\right) \prod_{s=b_{j}}^{b_{j-1}-1}\left((j-i) u+\left(s-a_{i-1}\right) u\right) \\
F_{P, Q}^{z}(u, v, t):=\prod_{\substack{1 \leq i \leq j \leq r \\
a_{j-1}-1}} \prod_{s=a_{j}}^{b_{j-1}-1}\left(1+z\left((i-j-1) u+\left(b_{i-1}-s-1\right) v+t\right)\right) \\
\prod_{s=b_{j}}\left(1+z\left((j-i) u+\left(s-a_{i-1}\right) v+t\right)\right)
\end{gathered}
$$

By lemma 3.2, when putting the correct weights $F_{P, Q}(u, v)$ will represent the top Chern class of $\operatorname{Hilb}^{d}(S \sqcup S)$ and $F_{P, Q}^{z}(u, v, t)$ the total Chern class of $\operatorname{Ext}_{q}^{1}\left(\mathcal{I}_{\mathcal{Z}_{1}}, \mathcal{I}_{\mathcal{Z}_{2}} \otimes p^{*} V\right)$.

Theorem 3.6. Let $\alpha_{1}, \ldots, \alpha_{N-2 r} \in H^{2}(S, \mathbb{Z})$. If $T$ is sufficiently general, then

$$
\begin{aligned}
& \delta_{\xi}\left(\alpha_{1} \alpha_{2} \ldots \alpha_{N-2 r} p t^{r}\right)=\operatorname{Coeff}_{z^{2 d}}\left(\sum_{\left(\left(P_{i}\right),\left(Q_{i}\right)\right) \in P_{2 m}(d)}\right. \\
& \left(\prod_{k=1}^{N-2 r}\left(\left\langle\xi, \alpha_{k}\right\rangle / 2+\sum_{i=1}^{m} w_{i}\left(\alpha_{k}\right)\left(n_{i}+m_{i}\right) z\right)\left(-1 / 4+\sum_{i=1}^{m} w_{i}\left(L_{1}\right) w_{i}\left(L_{2}\right)\left(n_{i}+m_{i}\right) z^{2}\right)^{r} .\right. \\
& \left(\prod _ { i = 1 } ^ { m } \left(\bar{F}_{\left(P_{i}, P_{i}\right)}\left(w\left(x_{i}\right), w\left(y_{i}\right)\right) \bar{F}_{Q_{i}, Q_{i}}\left(w\left(x_{i}\right), w\left(y_{i}\right)\right) \cdot\right.\right. \\
& \left.\left.\left.F_{P_{i}, Q_{i}}^{z}\left(w\left(x_{i}\right), w\left(y_{i}\right),-w_{i}(\xi)\right) F_{P_{i}, Q_{i}}^{z}\left(w\left(x_{i}\right), w\left(y_{i}\right),-w_{i}(\xi)\right)+w_{i}\left(K_{S}\right)\right)\right)^{-1}\right) .
\end{aligned}
$$

Proof. The Chern classes of $\mathcal{V}_{\xi}=\operatorname{Ext}_{q}^{1}\left(\mathcal{I}_{\mathcal{Z}_{1}}, \mathcal{I}_{\mathcal{Z}_{2}} \otimes p^{*} V\right)$ are the same as those of the virtual bundle $\mathcal{V}_{\xi}-H^{1}(S, V) \otimes \mathcal{O}_{T_{\xi}}$. Therefore the result just follows by putting together lemma 3.2, lemma 3.4 
and applying the Bott residue formula 2.12. Notice that $T$ is sufficiently general if none of the denominators vanish.

This formula can be implemented as a Maple program.

\section{The Donaldson invariants of the Projective Plane}

In this section we want to compute the $S U(2)$ - and the $S O(3)$-invariants of the projective plane $\mathbb{P}_{2}$ by first computing on the blowup $\widehat{\mathbb{P}}_{2}$ and then using the blowup formulas.

In order to get started we need the following easy result of Q2]:

Lemma 4.1. Let $S$ be a rational ruled surface, $F$ the class of a fibre and $E$ the class of a section. Fix $\left(c_{1}, c_{2}\right) \in H^{2}(S, \mathbb{Z}) \times H^{4}(S, \mathbb{Z})$ with $\left\langle c_{1} \cdot F\right\rangle=1$. Then, for all $\epsilon>0$ which are sufficiently small, we have $M_{F+\epsilon E}\left(c_{1}, c_{2}\right)=\emptyset$. In particular we get for $N:=4 c_{2}-c_{1}^{2}-3$ that $\Phi_{c_{1}, N}^{S, F+\epsilon E}=0$.

We will denote by $E$ the exceptional divisor on $\widehat{\mathbb{P}}_{2}$ and by $H$ the (pullback of) the hyperplane class on $\mathbb{P}_{2}$.

4.1. The $S U(2)$-case. We first consider the $S O(3)$-invariants on $\widehat{\mathbb{P}}_{2}$ with respect to Chern classes $\left(E, c_{2}\right)$ and put $N:=4 c_{2}-3$. For $0<\epsilon<<1$ the polarisation $L_{\epsilon}:=H-\epsilon E$ of $\widehat{\mathbb{P}}_{2}$ lies in a chamber of type $\left(E, c_{2}\right)$ which is related to the polarisation $H$ of $\mathbb{P}_{2}$. Thus 2.7.1) gives

$$
\Phi_{0, N}^{\mathbb{P}_{2}, H}\left(H^{N-2 r} p t^{r}\right)=\Phi_{E, N+1}^{\widehat{\mathbb{P}}_{2}, L_{\epsilon}}\left(\check{E} \check{H}^{N-2 r} p t^{r}\right) .
$$

On the other hand we know by lemma 4.1 that $\Phi_{E, N+1}^{\widehat{\mathbb{P}}_{2}, L_{1-\epsilon}}=0$, for $L_{1-\epsilon}:=H-(1-\epsilon) E$. Thus we get

$$
\Phi_{0, N}^{\mathbb{P}_{2}, H}\left(H^{N-2 r} p t^{r}\right)=\sum_{\xi \in W_{E, c_{2}}^{\widehat{\mathbb{P}}_{2}}(H-E, H)}(-1)^{e_{\xi, N+1}} \delta_{\xi, N+1}\left(\check{E} \check{H}^{N-2 r} p t^{r}\right),
$$

where $W_{E, c_{2}}^{\widehat{\mathbb{P}}_{2}}(H-E, H)$ is known by remark 2.10. Now we compute the $\delta_{\xi, N+1}\left(\check{E} \check{H}^{N-2 r} p t^{r}\right)$ with a maple program using the Bott residue theorem (i.e. theorem 3.6). For $N:=4 i+1$ we denote

$$
A_{N}:=\sum_{j=0}^{2 N} \Phi_{0, N}^{\mathbb{P}_{2}, H}\left(\check{H}^{N-2 j} p t^{j}\right) h^{N-2 j} p^{j} .
$$

Then our result is:

Theorem 4.2. The $S U(2)$-invariants of $\mathbb{P}_{2}$ are

$$
\begin{aligned}
& A_{1}=-\frac{3 h}{2}, A_{5}=h^{5}-p h^{3}-\frac{13 p^{2} h}{8}, A_{9}=3 h^{9}+\frac{15 p h^{7}}{4}-\frac{11 p^{2} h^{5}}{16}-\frac{141 p^{3} h^{3}}{64}-\frac{879 p^{4} h}{256}, \\
& A_{13}=54 h^{13}+24 p h^{11}+\frac{159 p^{2} h^{9}}{8}+\frac{51 p^{3} h^{7}}{16}-\frac{459 p^{4} h^{5}}{128}-\frac{1515 p^{5} h^{3}}{256}-\frac{36675 p^{6} h}{4096}, \\
& A_{17}=2540 h^{17}+694 p h^{15}+\frac{487 p^{2} h^{13}}{2}+\frac{2251 p^{3} h^{11}}{16}+\frac{2711 p^{4} h^{9}}{64}-\frac{5 p^{5} h^{7}}{16}-\frac{3355 p^{6} h^{5}}{256}-\frac{143725 p^{7} h^{3}}{8192}-\frac{850265 p^{8} h}{32768}, \\
& A_{21}=233208 h^{21}+45912 p h^{19}+10625 p^{2} h^{17}+3036 p^{3} h^{15}+\frac{41103 p^{4} h^{13}}{32}+\frac{1741 p^{5} h^{11}}{4}+\frac{5619 p^{6} h^{9}}{64}- \\
& \frac{20379 p^{7} h^{7}}{1024}-\frac{754141 p^{8} h^{5}}{16384}-\frac{904239 p^{9} h^{3}}{16384}-\frac{10504593 p^{10} h}{131072}, \\
& A_{25}=35825553 h^{25}+\frac{21975543 p h^{23}}{4}+\frac{15224337 p^{2} h^{21}}{16}+\frac{12159687 p^{3} h^{19}}{64}+\frac{11618625 p^{4} h^{17}}{256}+\frac{15077511 p^{5} h^{15}}{1024}+ \\
& \frac{19602561 p^{6} h^{13}}{4096}+\frac{20676279 p^{7} h^{11}}{16384}+\frac{11107665 p^{8} h^{9}}{65536}-\frac{28437201 p^{9} h^{7}}{262144}-\frac{169509159 p^{10} h^{5}}{1048576}-\frac{757633329 p^{11} h^{3}}{4194304}-\frac{4334081031 p^{12} h}{16777216} \\
& A_{29}=8365418914 h^{29}+1047342410 p h^{27}+\frac{1157569571 p^{2} h^{25}}{8}+\frac{357034013 p^{3} h^{23}}{16}+\frac{499796309 p^{4} h^{21}}{128}+ \\
& \frac{25506259 p^{5} h^{19}}{32}+\frac{423516455 p^{6} h^{17}}{2048}+\frac{245576651 p^{7} h^{15}}{4096}+\frac{537423737 p^{8} h^{13}}{32768}+\frac{118590907 p^{9} h^{11}}{32768}+\frac{131266019 p^{10} h^{9}}{524288}- \\
& \frac{498648655 p^{11} h^{7}}{1048576}-\frac{4800905323 p^{12} h^{5}}{8388608}-\frac{2551074181 p^{13} h^{3}}{4194304}-\frac{115237180987 p^{14} h}{134217728},
\end{aligned}
$$




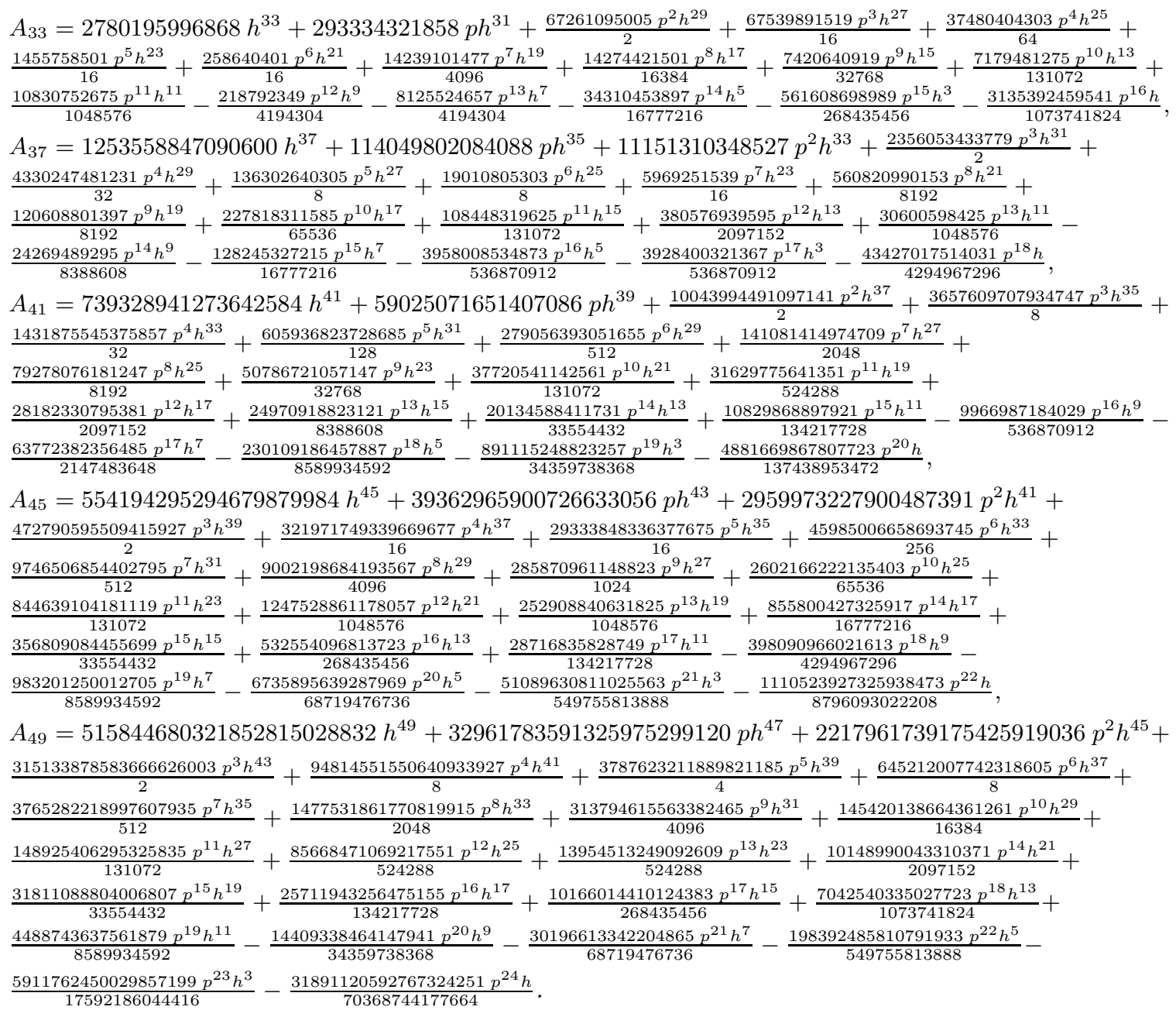

Remark 4.3. Note that the coefficients of the monomial $h^{N-2 j} p^{j}$ of $A_{N}$ are not well-defined for $j>(N-5) / 4$ because they do not lie in the stable range. We would like to thank Dieter Kotschick for pointing this out. One might however view the above formulas as a definition of these addititonal terms. One also sees that many of the invariants out of the stable range are negative whereas all those inside the stable range are positive (this was also pointed out to us by Dieter Kotschick).

4.2. The $S O(3)$-case. We consider first the $S O(3)$-invariants on $\widehat{\mathbb{P}}_{2}$ with respect to Chern classes $\left(H, c_{2}\right)$, and we put $N:=4 c_{2}-4$. For $0<\epsilon<<1$ the polarisation $L_{\epsilon}:=H-\epsilon E$ of $\widehat{\mathbb{P}}_{2}$ lies in a chamber of type $\left(H, c_{2}\right)$ related to the polarisation $H$ of $\mathbb{P}_{2}$. Thus 2.7.2) gives

$$
\Phi_{H, N}^{\mathbb{P}_{2}, H}\left(\check{H}^{N-2 r} p t^{r}\right)=\Phi_{H, N}^{\widehat{\mathbb{P}}_{2}, L_{\epsilon}}\left(\check{H}^{N-2 r} p t^{r}\right) .
$$

Putting $L_{1-\epsilon}:=H-(1-\epsilon) E$ we obtain $\Phi_{H}^{\widehat{\mathbb{P}}_{2}, L_{1-\epsilon}}\left(\check{H}^{N-2 r} p t^{r}\right)=0$. Thus we get

$$
\Phi_{H}^{\mathbb{P}_{2}, H}\left(\check{H}^{N-2 r} p t^{r}\right)=\sum_{\xi \in W_{H, c_{2}}^{\mathbb{\mathbb { P }}_{2}}(H-E, H)}(-1)^{e_{\xi, N}} \delta_{\xi, N}\left(\check{H}^{N-2 r} p t^{r}\right),
$$


and, using lemma 2.10, we can again carry out the computation with Botts formula. For $N:=4 i$ we denote

$$
B_{N}:=2^{2 i} \sum_{j=0}^{2 i} \Phi_{H}^{\mathbb{P}_{2}, H}\left(\check{H}^{N-2 j} p t^{j}\right) h^{N-2 j} p^{j} .
$$

Then we obtain:

Theorem 4.4. The $S O(3)$-Donaldson invariants of $\mathbb{P}_{2}$ are

$B_{0}=1, B_{4}=3 h^{4}+5 h^{2} p+19 p^{2}, B_{8} / 8=29 h^{8}+19 h^{6} p+17 h^{4} p^{2}+23 h^{2} p^{3}+85 p^{4}$,

$B_{12}=69525 h^{12}+26907 h^{10} p+12853 h^{8} p^{2}+7803 h^{6} p^{3}+6357 h^{4} p^{4}+8155 h^{2} p^{5}+29557 p^{6}$,

$B_{16} / 8=6231285 h^{16}+1659915 h^{14} p+519777 h^{12} p^{2}+194439 h^{10} p^{3}+88701 h^{8} p^{4}+51027 h^{6} p^{5}+$ $39753 h^{4} p^{6}+49519 h^{2} p^{7}+176837 p^{8}$,

$B_{20}=68081556995 h^{20}+13571675125 h^{18} p+3084569555 h^{16} p^{2}+808382629 h^{14} p^{3}+247407779 h^{12} p^{4}+$ $89811541 h^{10} p^{5}+39553139 h^{8} p^{6}+21987589 h^{6} p^{7}+16652099 h^{4} p^{8}+20329653 h^{2} p^{9}+71741715 p^{10}$, $B_{24} / 8=19355926872345 h^{24}+3046788353175 h^{22} p+535206161485 h^{20} p^{2}+105824308635 h^{18} p^{3}+$ $23774344785 h^{16} p^{4}+6132120911 h^{14} p^{5}+1838332965 h^{12} p^{6}+651103923 h^{10} p^{7}+279395017 h^{8} p^{8}+$ $151590087 h^{6} p^{9}+112496445 h^{4} p^{10}+135266955 h^{2} p^{11}+472659585 p^{12}$, $B_{28}=536625215902182969 h^{28}+69259301021976999 h^{26} p+9817859613586809 h^{24} p^{2}+$ $1538955926660199 h^{22} p^{3}+268722697637049 h^{20} p^{4}+52689438785319 h^{18} p^{5}+11702994789369 h^{16} p^{6}+$ $2974340336103 h^{14} p^{7}+875889126201 h^{12} p^{8}+304140743847 h^{10} p^{9}+127923966585 h^{8} p^{10}+$ $68135251815 h^{6} p^{11}+49776298425 h^{4} p^{12}+59127015975 h^{2} p^{13}+204876497145 p^{14}$, $B_{32} / 8=332465777488176686045 h^{32}+36176961518799287203 h^{30} p+4270943660527526777 h^{28} p^{2}+$ $550013108311246927 h^{26} p^{3}+77722220365607813 h^{24} p^{4}+12129004922528395 h^{22} p^{5}+$ $2104879834580993 h^{20} p^{6}+409294250544727 h^{18} p^{7}+89934657950957 h^{16} p^{8}+22556396083123 h^{14} p^{9}+$ $6542216760905 h^{12} p^{10}+2235172850335 h^{10} p^{11}+925169690645 h^{8} p^{12}+485534741275 h^{6} p^{13}+$ $350230091345 h^{4} p^{14}+411833933095 h^{2} p^{15}+1416634092797 p^{16}$, $B_{36}=17982292064097834276691197 h^{36}+1685376850354867108198203 h^{34} p+$ $169728914674713290425549 h^{32} p^{2}+18446964561578451602667 h^{30} p^{3}+2174127485943121961373 h^{28} p^{4}+$ $279319741333450241435 h^{26} p^{5}+39339602087475090285 h^{24} p^{6}+6111138005878747467 h^{22} p^{7}+$ $1054025359144892989 h^{20} p^{8}+203321142108471291 h^{18} p^{9}+44233113780975117 h^{16} p^{10}+$ $10964566444466603 h^{14} p^{11}+3139014782527197 h^{12} p^{12}+1058019835991643 h^{10} p^{13}+432158763674797 h^{8} p^{14}+$ $224042778598923 h^{6} p^{15}+159901382125437 h^{4} p^{16}+186411458197691 h^{2} p^{17}+637107121682253 p^{18}$, $B_{40} / 8=19983831593150830258093037499 h^{40}+1640698532032417214980201941 h^{38} p+$ $143617787626796457582947271 h^{36} p^{2}+13451663520190902994423761 h^{34} p^{3}+$ $1353428584063925323593987 h^{32} p^{4}+146907352128976242766365 h^{30} p^{5}+$ $17282999997688436388975 h^{28} p^{6}+2214864601846913417145 h^{26} p^{7}+310874334747308389131 h^{24} p^{8}+$ $48070219333713236901 h^{22} p^{9}+8241254396581767639 h^{20} p^{10}+1577751227160324321 h^{18} p^{11}+$ $340134212696649171 h^{16} p^{12}+83440287229631085 h^{14} p^{13}+23620292992955391 h^{12} p^{14}+$ $7869891016663881 h^{10} p^{15}+3178622918644059 h^{8} p^{16}+1630875748081269 h^{6} p^{17}+$ $1153440155417319 h^{4} p^{18}+1334613223327473 h^{2} p^{19}+4535236702668195 p^{20}$, $B_{44} / 8=226901192268190530686926956861797 h^{44}+16542462134525153318253326085835 h^{42} p+$ $1277706977403778580365852666661 h^{40} p^{2}+104862798979925329727378003659 h^{38} p^{3}+$ $9174416297780080293761973989 h^{36} p^{4}+858689743856030000767365835 h^{34} p^{5}+$ $86310585758469215596920485 h^{32} p^{6}+9355633875773319246298315 h^{30} p^{7}+$ $1098557533992391977544805 h^{28} p^{8}+$ 


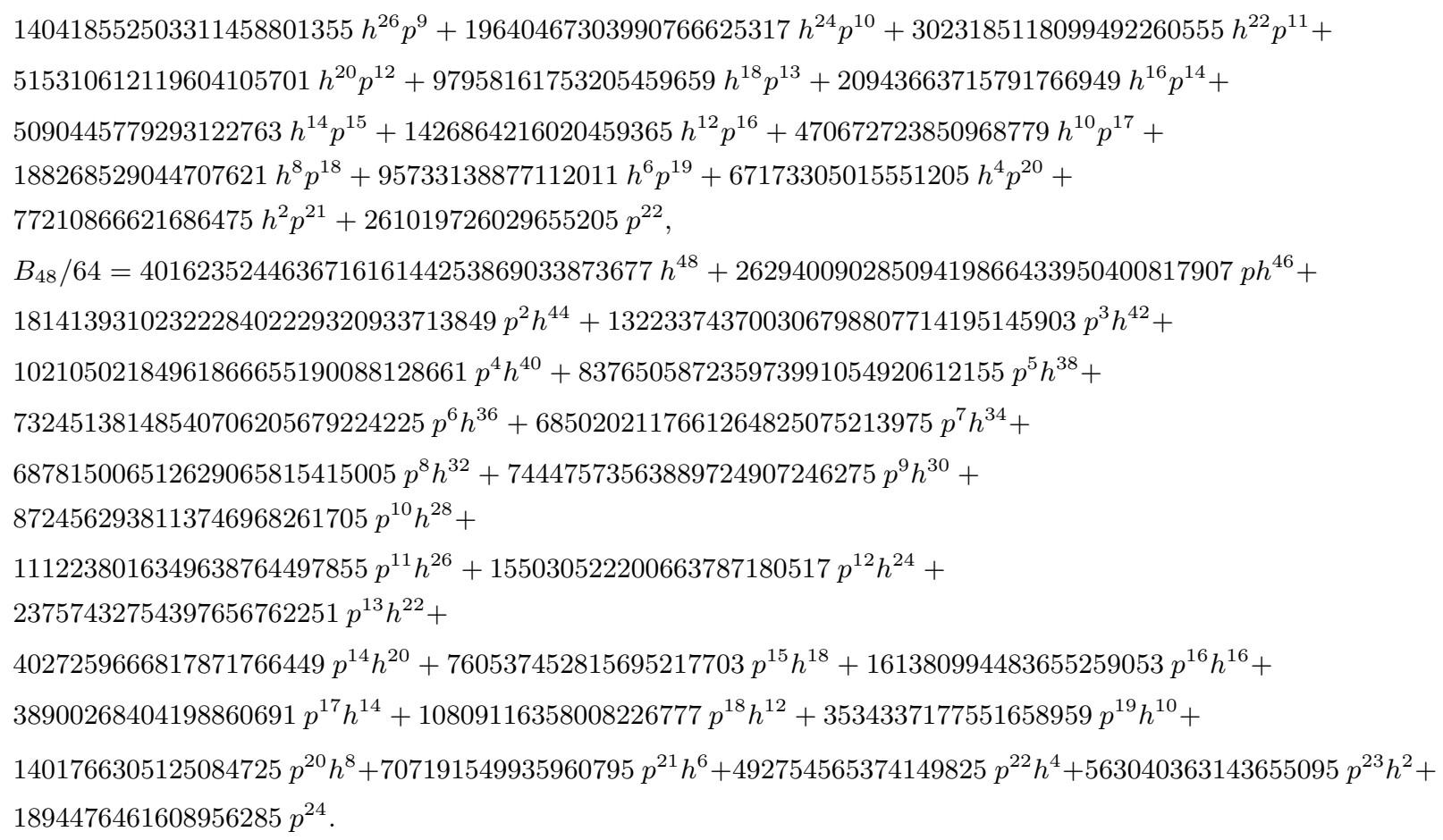

Conjecture 4.5. For all $n$ there is a nonnegative integer $l(n)$ such that $B_{4 n} / 2^{l(n)}$ is a polynomial in $h$ and $p$ all of whose coefficients are odd positive integers.

Remark 4.6. $B_{0}$ to $B_{16}$ were already computed in $\mathrm{K}-\mathrm{L}$ also using blowup and wall-crossing formulas, showing that $\mathbb{P}_{2}$ is not of simple type. Apart from slightly different conventions their results agree with ours. Their results and the computations of the $S U(2)$ invariants by various other authors have been quite useful to check the correctness of our programs - and thus of the computations in section 3 - in earlier stages of our work. The conjecture above could already have been made on the basis of their result.

\section{WALL-CROSSING FORMULAS}

In our paper [E-G] we formulated a conjecture about the shape of the wall-crossing formula, compatible with the conjecture of Kotschick and Morgan [K-M]. Here we state a slightly stronger form of the conjecture which is also supported by the computations in [E-G].

Conjecture 5.1. In the polynomial ring on $H^{2}(S, \mathbb{Q})$ we have

$$
\delta_{\xi, N, r}=(-4)^{-r} \sum_{k=0}^{d} \frac{(N-2 r) !}{(N-2 r-2 d+2 k) !(d-k) !} Q_{k}\left(N, d, r, K_{S}^{2}\right) L_{\xi / 2}^{N-2 r-2 d+2 k} q_{S}^{d-k}
$$

where $Q_{k}\left(N, d, r, K_{S}^{2}\right)$ is a polynomial of degree $k$ in $N, d, r, K_{S}^{2}$, which is independent of $S$ and $\xi$. 
We now will show, that, assuming the conjecture, we can compute several of the $Q_{k}\left(N, d, r, K_{S}^{2}\right)$. This computation will also give a check of the conjecture in many specific cases.

For all $i \geq 0$ we put

$P_{i}\left(N, d, r, K^{2}\right):=\left(\begin{array}{c}-2 d+2 N+2 K^{2}-24 r+\frac{13+3 i}{2} \\ i\end{array}\right)+(3 N-288 r)\left(\begin{array}{c}-2 d+2 N+2 K^{2}-24 r+\frac{7+3 i}{2} \\ i-2\end{array}\right)$.

Proposition 5.2. If conjecture 5.1 is true, then for $i=0,1,2,3,4$ we can write $Q_{i}\left(N, d, r, K^{2}\right)=$ $P_{i}\left(N, d, r, K^{2}\right)+R_{i}\left(N, d, r, K^{2}\right)$, where $R_{i}\left(N, d, r, K^{2}\right)=0$ for $i<2$ and

$R_{2}\left(N, d, r, K^{2}\right)=\frac{69}{8}$,

$R_{3}\left(N, d, r, K^{2}\right)=-13 d+29 N+17 K^{2}-5452 r+91$,

$R_{4}\left(N, d, r, K^{2}\right)=\frac{35 d^{2}}{4}-\frac{99 d N}{2}-\frac{51 d\left(K^{2}\right)}{2}+10802 d r+\frac{181 N^{2}}{4}+\frac{115 N\left(K^{2}\right)}{2}-12050 N r+\frac{67\left(K^{2}\right)^{2}}{4}-$ $10898\left(K^{2}\right) r+169836 r^{2}-\frac{537 d}{4}+\frac{2821 N}{8}+\frac{761\left(K^{2}\right)}{4}-146495 r+\frac{72005}{128}$.

Proof. We assume conjecture 5.1. Let $X=\mathbb{P}_{1} \times \mathbb{P}_{1}$ or a blow up of $\mathbb{P}_{1} \times \mathbb{P}_{1}$ in finitely many points. We denote by $F$ and $G$ the pullbacks of the fibres of the two projections from $\mathbb{P}_{1} \times \mathbb{P}_{1}$ to $\mathbb{P}_{1}$. For a class $\xi=F-s G$ in $H^{2}(X, \mathbb{Z})$ defining a wall and an integer $d \geq 0$, let $N:=4 d+2 s-3$. Then on $X$ we can determine the coefficients $a_{k}$ of $L_{\xi / 2}^{N-2 r-2 d+2 k} q_{X}^{d-k}$ in $\delta_{\xi, N, r}$ as follows: We can assume that $X$ has an action of $\mathbb{C}^{*}$ with finitely many fixpoints as in 3.1. For $x$ an indeterminant we put $\alpha:=-x F+G$ and compute the polynomial $\delta_{\xi, N, r}\left(\alpha^{N-2 r}\right)$ in $x$ using the Bott residue formula on $\operatorname{Hilb}^{d}(X \sqcup X)$, from which we can compute the $a_{k}$.

Now we can compute the polynomials $Q_{k}\left(N, d, r, K_{X}^{2}\right)$ as follows: We consider all nonnegative integers $d, w, b, r$ with $d \geq k$ and $d+w+b+r \leq 2 k$. Let $X$ be the blow up of $\mathbb{P}_{1} \times \mathbb{P}_{1}$ in $b$ points. With the method of the last paragraph we compute the coefficient $c_{d, w, b, r}$ of $L_{\xi / 2}^{N-2 r-2 d+2 k} q_{X}^{d-k}$ in $\delta_{\xi, N, r}$ on $X$, where $N:=4 d+2 w+1$ and $\xi=F-(w+2) G$. Using all the $c_{d, w, b, r}$ we obtain a system of $\left(\begin{array}{c}k+4 \\ 4\end{array}\right)$ linear equations for the coefficients of the $N^{i} d^{j} r^{t}\left(K_{S}^{2}\right)^{s}$ (with $0 \leq i+j+t+s \leq k$ ) in $Q_{k}\left(N, d, r, K_{S}^{2}\right)$. Solving this system of equations we obtain our result. All the computations are again carried out using a suitable Maple program.

The formulas suggest the following conjecture:

Conjecture 5.3. $\quad$ 1. For all $i$ the polynomial $Q_{i}\left(N, d, r, K^{2}\right)$ is of the form $Q_{i}\left(N, d, r, K^{2}\right)=$ $P_{i}\left(N, d, r, K^{2}\right)+R_{i}\left(N, d, r, K^{2}\right)$, where $R_{i}\left(N, d, r, K^{2}\right)$ is a polynomial in $N, d, r, K^{2}$ of degree $i-2$.

2. If we view $R_{i}\left(N, d, r, K^{2}\right)$ as a polynomial in $N,-d,-r, K^{2}$, then all its coefficients are positive and the same is true for $Q_{i}\left(N, d, r, K^{2}\right)$.

Proposition 5.4. If conjecture 5.1 and part (1) of conjecture 5.3 are true then $R_{5}\left(N, d, r, K^{2}\right)=11\left(K^{2}\right)^{3}+57\left(K^{2}\right)^{2} N-25\left(K^{2}\right)^{2} d-10892\left(K^{2}\right)^{2} r+90\left(K^{2}\right) N^{2}-98\left(K^{2}\right) N d-$ $24088\left(K^{2}\right) N r+17\left(K^{2}\right) d^{2}+21592\left(K^{2}\right) d r+339600\left(K^{2}\right) r^{2}+44 N^{3}-82 N^{2} d-13304 N^{2} r+$ $41 N d^{2}+23896 N d r+363792 N r^{2}-3 d^{3}-10700 d^{2} r-338448 d r^{2}-2525760 r^{3}+198\left(K^{2}\right)^{2}+$ $744\left(K^{2}\right) N-276\left(K^{2}\right) d-303600\left(K^{2}\right) r+618 N^{2}-612 N d-333888 N r+78 d^{2}+301008 d r+$ $5457888 r^{2}+1213\left(K^{2}\right)+2506 N-729 d-3101884 r+2490$, 


$$
\begin{aligned}
& R_{6}\left(N, d, r, K^{2}\right)=\frac{65\left(K^{2}\right)^{4}}{12}+\frac{113\left(K^{2}\right)^{3} N}{3}-\frac{49\left(K^{2}\right)^{3} d}{3}-\frac{21772\left(K^{2}\right)^{3} r}{3}+\frac{179\left(K^{2}\right)^{2} N^{2}}{2}-97\left(K^{2}\right)^{2} N d- \\
& 24076\left(K^{2}\right)^{2} N r+\frac{33\left(K^{2}\right)^{2} d^{2}}{2}+21580\left(K^{2}\right)^{2} d r+339528\left(K^{2}\right)^{2} r^{2}+\frac{263\left(K^{2}\right) N^{3}}{3}-163\left(K^{2}\right) N^{2} d- \\
& 26596\left(K^{2}\right) N^{2} r+81\left(K^{2}\right) N d^{2}+47768\left(K^{2}\right) N d r+727440\left(K^{2}\right) N r^{2}-\frac{17\left(K^{2}\right) d^{3}}{3}-21388\left(K^{2}\right) d^{2} r- \\
& 676752\left(K^{2}\right) d r^{2}-5050944\left(K^{2}\right) r^{3}+\frac{365 N^{4}}{12}-\frac{247 N^{3} d}{3}-\frac{29332 N^{3} r}{3}+\frac{147 N^{2} d^{2}}{2}+26404 N^{2} d r+389208 N^{2} r^{2}- \\
& \frac{65 N d^{3}}{3}-23692 N d^{2} r-725136 N d r^{2}-5327424 N r^{3}+\frac{d^{4}}{12}+\frac{21196 d^{3} r}{3}+337224 d^{2} r^{2}+5041728 d r^{3}+ \\
& 24147648 r^{4}+\frac{821\left(K^{2}\right)^{3}}{6}+\frac{3127\left(K^{2}\right)^{2} N}{4}-\frac{565\left(K^{2}\right)^{2} d}{2}-314198\left(K^{2}\right)^{2} r+1306\left(K^{2}\right) N^{2}-\frac{2567\left(K^{2}\right) N d}{2}- \\
& 691258\left(K^{2}\right) N r+\frac{309\left(K^{2}\right) d^{2}}{2}+623020\left(K^{2}\right) d r+11250984\left(K^{2}\right) r^{2}+\frac{7987 N^{3}}{12}-1154 N^{2} d-380192 N^{2} r+ \\
& \frac{2007 N d^{2}}{4}+685594 N d r+12076284 N r^{2}-\frac{53 d^{3}}{6}-308822 d^{2} r-11204904 d r^{2}-93985056 r^{3}+\frac{249499\left(K^{2}\right)^{2}}{192}+ \\
& \frac{529411\left(K^{2}\right) N}{96}-\frac{48265\left(K^{2}\right) d}{32}-\frac{156136081\left(K^{2}\right) r}{24}+\frac{944107 N^{2}}{192}-\frac{134657 N d}{32}-\frac{170651209 N r}{24}+\frac{41627 d^{2}}{192}+\frac{154931089 d r}{24}+ \\
& \frac{538005841 r^{2}}{4}+\frac{1027343\left(K^{2}\right)}{192}+\frac{5033035 N}{384}-\frac{406799 d}{192}-\frac{1032193219 r}{16}+\frac{7872921}{1024} .
\end{aligned}
$$

Proof. The method of the proof is very similar to that of proposition 5.2. Using conjecture 5.3 we can reduce the number of equations of the linear system we have to solve. For the computation of $R_{k}$ we have in the notations of the proof of proposition 5.2 only to consider $d, w, b, r$ with $d \geq k$ and $d+w+b+r \leq 2 k-2$.

Proposition 5.5. Assume conjecture 5.1. Then for $d \leq 8$ and $K^{2}=8$ also conjecture 5.5 holds. Furthermore we have

$R_{7}(N, 7, r, 8)=\frac{242 N^{5}}{15}-\frac{16136 N^{4} r}{3}+277280 N^{3} r^{2}-5613696 N^{2} r^{3}+50448384 N r^{4}-\frac{843153408 r^{5}}{5}+$ $584 N^{4}-\frac{926104 N^{3} r}{3}+14133648 N^{2} r^{2}-215219520 N r^{3}+1068715008 r^{4}+\frac{49259 N^{3}}{6}-\frac{27036430 N^{2} r}{3}+$ $327411912 N r^{2}-2834114784 r^{3}+55637 N^{2}-\frac{491711972 N r}{3}+3453170368 r^{2}+\frac{5432731 N}{30}-\frac{7607891522 r}{5}+$ 226878

$R_{7}(N, 8, r, 8)=\frac{242 N^{5}}{15}-\frac{16136 N^{4} r}{3}+277280 N^{3} r^{2}-5613696 N^{2} r^{3}+50448384 N r^{4}-\frac{843153408 r^{5}}{5}+$ $526 N^{4}-\frac{867848 N^{3} r}{3}+13357680 N^{2} r^{2}-204584256 N r^{3}+1020478464 r^{4}+\frac{40547 N^{3}}{6}-\frac{24608014 N^{2} r}{3}+$ $301879416 N r^{2}-2636445408 r^{3}+\frac{85365 N^{2}}{2}-\frac{444885076 N r}{3}+3161672456 r^{2}+\frac{1989263 N}{15}-\frac{6895690692 r}{5}+$ 162324 ,

$R_{8}(N, 8, r, 8)=\frac{69 N^{6}}{10}-\frac{35428 N^{5} r}{15}+147976 N^{4} r^{2}-3940224 N^{3} r^{3}+52663680 N^{2} r^{4}-\frac{1749924864 N r^{5}}{5}+$ $\frac{4618156032 r^{6}}{5}+\frac{17993 N^{5}}{60}-164166 N^{4} r+9819288 N^{3} r^{2}-222171264 N^{2} r^{3}+2193801408 N r^{4}-\frac{39855794688 r^{5}}{5}+$ $\frac{342159 N^{4}}{64}-\frac{75235799 N^{3} r}{12}+\frac{675173309 N^{2} r^{2}}{2}-5810679060 N r^{3}+32006797596 r^{4}+\frac{9540439 N^{3}}{192}-\frac{678142435 N^{2} r}{4}+$ $\frac{28388710281 N r^{2}}{4}-68700098862 r^{3}+\frac{646487951 N^{2}}{2560}-\frac{602240211743 N r}{192}+\frac{11796298005871 r^{2}}{160}+\frac{10191068747 N}{15360}-$ $\frac{19195182347591 r}{640}+\frac{23061793325}{32768}$.

Proof. The method is again similar to that of the proof of proposition 5.2. Now we carry out our computations on $X=\mathbb{P}_{1} \times \mathbb{P}_{1}$. In the notation of the proof of proposition 5.2 we have therefore $b=0$. For the computation of $R_{k}$ we consider nonnegative integers $d, w, r$ with $d+w+r \leq 2 k$ and $k \leq d \leq 8$.

Proposition 5.6. Let $S$ be a rational ruled surface, then for $N \leq 40$ and $d \leq 8$ the conjectures 5.1 and 5.3 are correct (and therefore also all the formulas above). 
Proof. Any rational ruled surface $X$ is a degeneration of either or $\mathbb{P}_{1} \times \mathbb{P}_{1}$ or $\widehat{\mathbb{P}}_{2}$, and under the degeneration the ample cone of $X$ corresponds to a subcone of the ample cone of $\mathbb{P}_{1} \times \mathbb{P}_{1}$ (resp. $\widehat{\mathbb{P}}_{2}$ ). Therefore it is enough to prove the result for $\mathbb{P}_{1} \times \mathbb{P}_{1}$ and $\widehat{\mathbb{P}}_{2}$. We let $c_{1}$ run through $0, F, G, F+G$ on $\mathbb{P}_{1} \times \mathbb{P}_{1}$ and through $0, H, E, F$ on $\widehat{\mathbb{P}}_{2}(F=H-E)$. For $S=\mathbb{P}_{1} \times \mathbb{P}_{1}$ and $S=\widehat{\mathbb{P}}_{2}$ we consider for all integers $d$ with $0 \leq d \leq 8$ the set $W_{S, d}$ of all classes $\xi$, which define a wall of type $\left(c_{1}, c_{2}\right)$, such that $N:=4 d-\xi^{2}-3 \leq 40$ and $\langle\xi \cdot F\rangle<0$. It is easy to see that

$$
\begin{aligned}
W_{\mathbb{P}_{1} \times \mathbb{P}_{1}, d} & =\{\xi=a F-b G \mid a>0, b>0,2 a b \leq 40-4 d+3\}, \\
W_{\widehat{\mathbb{P}}_{2}, d} & =\left\{\xi=b H-a E \mid a>b>0, a^{2}-b^{2} \leq 40-4 d+3\right\} .
\end{aligned}
$$

For all $d \leq 8$ and all $\xi \in W_{S, d}$ we again compute all the coefficients of $L_{\xi / 2}^{N-2 r-2 d+2 k} q_{S}^{d-k}$ with the method of the first paragraph of the proof of proposition 5.2 .

\section{The Donaldson Invariants of Birationally Ruled surfaces.}

In this section we will show that our algorithm for computing the wall-crossing formula $\delta_{\xi, N}$ and the blowup formulas enable us to compute all the Donaldson invariants of all rational surfaces $X$ for all generic polarisations lying in a suitable subcone of the ample cone of $X$.

6.1. The case of rational ruled surfaces. In this case we can indeed determine the Donaldson invariants for all generic polarisations. For simplicity we will only compute the restriction of the Donaldson invariants to $\operatorname{Sym}^{N}\left(H_{2}(S, \mathbb{Q})\right)$. In $\left[\mathrm{K}-\mathrm{L}\right.$ some invariants of $\mathbb{P}_{1} \times \mathbb{P}_{1}$ have been computed also using blowup and wall-crossing formulas. Their results show e.g. that there is no chamber, for which $\mathbb{P}_{1} \times \mathbb{P}_{1}$ is of simple type. Our results again agree with theirs and earlier results e.g. in $[\mathrm{L}-\mathrm{Q}$.

Theorem 6.1. Let $S$ be a rational ruled surface, $F$ the class of a fibre and $q_{S}$ the quadratic form on $H_{2}(S, \mathbb{Z})$. We denote by $F_{\epsilon}$ the polarisation $F+\epsilon E$, where $E$ is the class of a section with nonpositive selfintersection.

(1) For $\epsilon>0$ sufficiently small we have $\Phi_{E, N}^{S, F_{\epsilon}}=0$ and $\Phi_{E+F, N}^{S, F_{\epsilon}}=0$.

(2) For $\epsilon>0$ sufficiently small we have for $E_{N}:=\Phi_{0, N, 0}^{S, F_{\epsilon}}$ :

$E_{5}=-L_{F}^{5}+5 / 2 L_{F}^{3} q_{S}-5 / 2 L_{F} q_{S}^{2}$,

$E_{9}=40 L_{F}^{9}-108 L_{F}^{7} q_{S}+108 L_{F}^{5} q_{S}^{2}-42 L_{F}^{3} q_{S}^{3}$,

$E_{13}=-9345 L_{F}^{13}+26949 L_{F}^{11} q_{S}-31590 L_{F}^{9} q_{S}^{2}+18018 L_{F}^{7} q_{S}^{3}-4290 L_{F}^{5} q_{S}^{4}$,

$E_{17}=7369656 L_{F}^{17}-22136040 L_{F}^{15} q_{S}+28474320 L_{F}^{13} q_{S}^{2}-19734960 L_{F}^{11} q_{S}^{3}+7425600 L_{F}^{9} q_{S}^{4}-1225224 L_{F}^{7} q_{S}^{5}$,

$E_{21}=-14772820744 L_{F}^{21}+45586042992 L_{F}^{19} q_{S}-62181472500 L_{F}^{17} q_{S}^{2}+48231175860 L_{F}^{15} q_{S}^{3}-$

$22562971200 L_{F}^{13} q_{S}^{4}+6074420688 L_{F}^{11} q_{S}^{5}-740703600 L_{F}^{9} q_{S}^{6}$,

$E_{25}=63124363433664 L_{F}^{25}-198545836440000 L_{F}^{23} q_{S}+281925714232800 L_{F}^{21} q_{S}^{2}-235199340734400 L_{F}^{19} q_{S}^{3}+$

$125056219068000 L_{F}^{17} q_{S}^{4}-42588214875360 L_{F}^{15} q_{S}^{5}+8649138960000 L_{F}^{13} q_{S}^{6}-813136737600 L_{F}^{11} q_{S}^{7}$,

$E_{29}=-509894102555251905 L_{F}^{29}+1626742370158553130 L_{F}^{27} q_{S}-2378321090933081112 L_{F}^{25} q_{S}^{2}+$

$2087846466793743600 L_{F}^{23} q_{S}^{3}-1207966082767844400 L_{F}^{21} q_{S}^{4}+473530658232013200 L_{F}^{19} q_{S}^{5}-$

$123363365393268000 L_{F}^{17} q_{S}^{6}+19623703009790880 L_{F}^{15} q_{S}^{7}-1467326424564000 L_{F}^{13} q_{S}^{8}$,

$E_{33}=7135482220088837442520 L_{F}^{33}-23016295766978863295760 L_{F}^{31} q_{S}+34404291587748659734080 L_{F}^{29} q_{S}^{2}-$ 
$31360607908598315276160 L_{F}^{27} q_{S}^{3}+19266231547036209415680 L_{F}^{25} q_{S}^{4}-8299005150626510918400 L_{F}^{23} q_{S}^{5}+$ $2515398487826672448000 L_{F}^{21} q_{S}^{6}-519339581441771650560 L_{F}^{19} q_{S}^{7}+66567414222758592000 L_{F}^{17} q_{S}^{8}-$ $4055565288690115200 L_{F}^{15} q_{S}^{9}$.

(3) For $\epsilon>0$ sufficiently small and all $N \leq 33$ we have, writing $\Phi_{0, N, 0}^{S, F_{\epsilon}}$ as a polynomial $E_{N}\left(L_{F}, q_{S}\right)$ in $L_{F}$ and $q_{S}$,

$$
\Phi_{F, N, 0}^{S, F_{\epsilon}}=E_{N}\left(L_{F}, q_{S}\right)-E_{N}\left(L_{F} / 2, q_{S}\right) .
$$

Proof. (1) is just lemma 4.1 .

(2) and (3): We fix $N:=4 c_{2}-3$ with $c_{2}>1$. We will just compute the corresponding Donaldson invariants explicitely. As any pair $(S, L)$ consisting of a Hirzebruch surface $S=\Sigma_{n}$ and $L=$ $a F+b E \in \operatorname{Pic}(S)$ (where $E$ is a section with selfintersection $-n \leq 0$ ) can be deformed to either $\left(\mathbb{P}_{1} \times \mathbb{P}_{1}, a F+b(E-n F / 2)\right)$ or $\left(\widehat{\mathbb{P}}_{2}, a F+b(E-(n-1) F / 2)\right)$ we see that we can assume that $S=\mathbb{P}_{1} \times \mathbb{P}_{1}$ or $S=\widehat{\mathbb{P}}_{2}$ and $c_{1}=F$ or $c_{1}=0$.

(a) $S=\mathbb{P}_{1} \times \mathbb{P}_{1}, c_{1}=F$ (we will always denote by $F$ and $G$ the fibres of the projections to the two factors). By (1) we have for $\epsilon>0$ sufficiently small $\Phi_{F, N}^{\mathbb{P}_{1} \times \mathbb{P}_{1}, G+\epsilon F}=0$. Therefore we get

$$
\Phi_{F, N}^{\mathbb{P}_{1} \times \mathbb{P}_{1}, F_{\epsilon}}=-\sum_{\xi \in W_{F, c_{2}}^{\mathbb{P}_{1} \times \mathbb{P}_{1}}(F, G)}(-1)^{e_{\xi, N}} \delta_{\xi, N} .
$$

So the invariants can be computed using proposition 5.6 and remark 2.10 .

(b) $S=\widehat{\mathbb{P}}_{2}, c_{1}=F$. Let $\epsilon>0$ be sufficiently small. By the $S O(3)$-blow up formula we have for all $\alpha \in A_{N-i}\left(\mathbb{P}_{2}\right)$ :

$$
\Phi_{F, N}^{\widehat{\mathbb{P}}_{2}, H-\epsilon E}\left(\check{E}^{i} \alpha\right)=\Phi_{H}^{\mathbb{P}_{2}, H}\left(S_{i}(p t) \alpha\right),
$$

and the $S O(3)$-invariants of $\mathbb{P}_{2}$ have been determined in theorem 4.4. Therefore

$$
\Phi_{F, N}^{\widehat{\mathbb{P}}_{2}, F_{\epsilon}}\left(\check{E}^{i} \alpha\right)=\Phi_{H}^{\mathbb{P}_{2}, H}\left(S_{i}(p t) \alpha\right)-\sum_{\xi \in W_{F, c_{2}}^{\widehat{\mathbb{P}}_{2}}(F, H)}(-1)^{e_{\xi, N}} \delta_{\xi, N}\left(\check{E}^{i} \alpha\right),
$$

So the sum can be computed using proposition 5.6 and remark 2.10.

(c) $S=\widehat{\mathbb{P}}_{2}, c_{1}=0$. Let $\widetilde{\mathbb{P}}_{2}$ be the blowup of $\mathbb{P}_{2}$ in two points with exceptional divisors $E_{1}$ and $E_{2}$. Then $\widetilde{\mathbb{P}}_{2}$ is also the blow up of $\mathbb{P}_{1} \times \mathbb{P}_{1}$ in a point. We denote the exceptional divisor by $E$. We denote by $F$ the pullback of $F=H-E_{1}$ from $\widehat{\mathbb{P}}_{2}$ (which coincides with the pullback of $F$ from $\left.\mathbb{P}_{1} \times \mathbb{P}_{1}\right)$. We have $F=E_{2}+E$. We also denote by $G$ the pullback of $G$ from $\mathbb{P}_{1} \times \mathbb{P}_{1}$ and have $G=E_{1}+E$. For $1>>\epsilon>>\mu>0$, let $H_{1}:=F+\epsilon G-\mu E$ and $H_{2}:=F+\epsilon G-(\epsilon-\mu) E$. Then $H_{2}$ is a polarisation of $\widetilde{\mathbb{P}}_{2}$ which lies in a $\left(E_{2}, c_{2}\right)$-chamber related to the $\left(0, c_{2}\right)$-chamber of $F+\epsilon E_{1}$ on $\widehat{\mathbb{P}}_{2}$. Thus by the $S O(3)$-blowup formula we have

$$
\Phi_{0, N}^{\widehat{\mathbb{P}}_{2}, F+\epsilon E_{1}}\left(\check{F}^{i} \check{E}_{1}^{N-i}\right)=-\Phi_{E_{2}, N+1}^{\widetilde{\mathbb{P}}_{2}, H_{2}}\left(\check{E}_{2} \check{F}^{i}(\check{G}-\check{E})^{N-i}\right)=-\Phi_{F-E, N+1}^{\widetilde{\mathbb{P}}_{2}, H_{2}}\left((\check{F}-\check{E}) \check{F}^{i}(\check{G}-\check{E})^{N-i}\right) .
$$

We have

$$
\Phi_{F-E, N+1}^{\widetilde{\mathbb{P}}_{2}, H_{1}}-\Phi_{F-E, N+1}^{\widetilde{\mathbb{P}}_{2}, H_{2}}=\sum_{\xi \in W_{F-E, c_{2}}^{\tilde{\mathbb{P}}_{2}}\left(H_{2}, H_{1}\right)}(-1)^{e_{\xi, N+1}} \delta_{\xi, N+1},
$$

and for $\epsilon$ sufficiently small is is easy to see that

$$
W_{F-E, c_{2}}^{\widetilde{\mathbb{P}}_{2}}\left(H_{2}, H_{1}\right)=\left\{(2 a-1) F-(2 b-1) E \mid b>a>0, b(b-1) \leq c_{2}\right\} .
$$


So $\Phi_{F-E, N+1}^{\widetilde{\mathbb{P}}_{2}, H_{1}}-\Phi_{F-E, N+1}^{\widetilde{\mathbb{P}}_{2}, H_{2}}$ can be computed by the Bott residue formula. Finally $H_{1}$ lies in a $\left(F-E, c_{2}\right)$-chamber on $\widetilde{\mathbb{P}}_{2}$ related to the $\left(F, c_{2}\right)$-chamber of $F+\epsilon G$ on $\mathbb{P}_{1} \times \mathbb{P}_{1}$. So, by the $S O(3)$ blowup formula (with exceptional divisor $E$ ), we get for $\alpha \in A_{N+1-i}\left(\mathbb{P}_{1} \times \mathbb{P}_{1}\right)$

$$
\Phi_{F-E, N+1}^{\widetilde{\mathbb{P}}_{2}, H_{1}}\left(\alpha \check{E}^{i}\right)=\Phi_{F}^{\mathbb{P}_{1} \times \mathbb{P}_{1}, F+\epsilon E}\left(\alpha S_{i}(p t)\right),
$$

and the last is computed by the method of (a). Now we put everything together to get our result.

(d) $S=\mathbb{P}_{1} \times \mathbb{P}_{1}, c_{1}=0$. This case is very similar to (c), only with the role of $\mathbb{P}_{1} \times \mathbb{P}_{1}$ and $\widehat{\mathbb{P}}_{2}$ exchanged. We use the same notations as in (c). Now $H_{1}$ is a polarisation of $\widetilde{\mathbb{P}}_{2}$ which lies in a $\left(E, c_{2}\right)$-chamber related to the $\left(0, c_{2}\right)$-chamber of $F+\epsilon G$ on $\mathbb{P}_{1} \times \mathbb{P}_{1}$. Thus by the $S O(3)$-blowup formula we have

$$
\Phi_{0, N}^{\mathbb{P}_{1} \times \mathbb{P}_{1}, F+\epsilon G}\left(\check{F}^{i} \check{G}^{N-i}\right)=-\Phi_{E, N+1}^{\widetilde{\mathbb{P}}_{2}, H_{1}}\left(\check{E} \check{F}^{i} \check{G}^{N-i}\right)=-\Phi_{F-E_{2}, N+1}^{\widetilde{\mathbb{P}}_{2}, H_{1}}\left(\left(\check{F}-\check{E}_{2}\right) \check{F}^{i}\left(\check{F}+\check{E}_{1}-\check{E}_{2}\right)^{N-i}\right) .
$$

We have

$$
\Phi_{E, N+1}^{\widetilde{\mathbb{P}}_{2}, H_{1}}-\Phi_{E, N+1}^{\widetilde{\mathbb{P}}_{2}, H_{2}}=\sum_{\xi \in W_{E, c_{2}}^{\widetilde{\mathbb{P}}_{2}}\left(H_{2}, H_{1}\right)}(-1)^{e_{\xi, N+1}} \delta_{\xi, N+1},
$$

and for $\epsilon$ sufficiently small is is easy to see that

$$
W_{E, c_{2}}^{\widetilde{\mathbb{P}}_{2}}\left(H_{2}, H_{1}\right)=\left\{\left(2 a F-(2 b-1) E \mid b-1 / 2>a>0, b(b-1) \leq c_{2}\right\} .\right.
$$

So $\Phi_{E, N+1}^{\widetilde{\mathbb{P}}_{2}, H_{1}}-\Phi_{E, N+1}^{\widetilde{\mathbb{P}}_{2}, H_{2}}$ can be computed by the Bott residue formula. Finally $H_{2}$ lies in a $\left(E, c_{2}\right)$ chamber on $\widetilde{\mathbb{P}}_{2}$ related to the $\left(F, c_{2}\right)$-chamber of $F+\epsilon E_{1}$ on $\widehat{\mathbb{P}}_{2}$. So, by the $S O(3)$-blowup formula (with exceptional divisor $E_{2}$ ), we get for $\alpha \in A_{N+1-i}\left(\widehat{\mathbb{P}}_{2}\right)$

$$
\Phi_{E, N+1}^{\widetilde{\mathbb{P}}_{2}, H_{2}}\left(\alpha \check{E}_{2}^{i}\right)=\Phi_{F}^{\widehat{\mathbb{P}}_{2}, F+\epsilon E}\left(\alpha S_{i}(p t)\right)
$$

and the last is computed by the method of (b).

Conjecture 6.2. For $S$ a rational ruled surface we have in the notation of theorem 6.1 for all $N=4 c_{2}-3$ with $c_{2} \geq 2$ :

1. $\Phi_{0, N, 0}^{S, F_{\epsilon}}$ and $\Phi_{F, N, 0}^{S, F_{\epsilon}}$ are polynomials $E_{0, N}\left(L_{F}, q_{S}\right)$ and $E_{F, N}\left(L_{F}, q_{S}\right)$ in $L_{F}$ and $q_{S}$, which are independent of $S$.

2. $E_{0, N}\left(L_{F}, q_{S}\right)$ and $E_{F, N}\left(L_{F}, q_{S}\right)$ are divisible by $L_{F}^{N-2 c_{2}}$.

3. $E_{F, N}\left(L_{F}, q_{S}\right)=E_{0, N}\left(L_{F}, q_{S}\right)-E_{0, N}\left(L_{F} / 2, q_{S}\right)$.

Remark 6.3. We keep the notation of theorem 6.1. Notice that theorem 6.1 and proposition 5.6 determines all the $S U(2)$ - and $S O(3)$ - Donaldson invariants of a rational ruled surface $S$ of degree at most 35 for all generic polarisations: Fix $\left(c_{1}, c_{2}\right)$ and put $N:=4 c_{2}-c_{1}^{2}-3$. If $L$ is a generic polarisation then

$$
\Phi_{c_{1}, N}^{L, S}=\Phi_{c_{1}, N}^{F_{\epsilon}, S}+\sum_{\xi \in W_{c_{1}, c_{2}}^{S}(F, L)}(-1)^{e_{\xi, N}} \delta_{\xi, N} .
$$

This sum is given for $N \leq 35$ by theorem 6.1, remark 2.10 and proposition 5.6 . 
6.2. The Donaldson invariants of blowups of $\mathbb{P}_{2}$. We want to finish by showing that our methods give an algorithm for computing all the Donaldson invariants for all rational surfaces $X$ at least for polarisations lying in a reasonably big subcone $C^{g}$ of the ample cone $C_{X}$ of $X$. In [K-L] it is shown that the Donaldson invariants of $\mathbb{P}_{2}$ and $\mathbb{P}_{1} \times \mathbb{P}_{1}$ can be determined from the wall-crossing formulas on some blowups, and our results can be seen as a generalization of this.

A rational surface $X$, which is neither $\mathbb{P}_{2}$ nor ruled can be deformed to a a blowup $\mathbb{P}_{2}\left(x_{1}, \ldots x_{r}\right)$ of $\mathbb{P}_{2}$ in finitely many general points. Under this deformation $C_{X}$ corresponds to a (in general strict) subcone of the ample cone $C_{\mathbb{P}_{2}\left(x_{1}, \ldots x_{r}\right)}$. We can therefore restrict our attention to $X=\mathbb{P}_{2}\left(x_{1}, \ldots x_{r}\right)$.

Theorem 6.4. There exists an algorithm computing all the $S U(2)$ - and $S O(3)$-Donaldson invariants of $\mathbb{P}_{2}\left(x_{1}, \ldots x_{r}\right)$ with respect to all generic polarisations in a nonempty open subcone $C^{g}$ of the ample cone of $\mathbb{P}_{2}\left(x_{1}, \ldots x_{r}\right)$.

Proof. Let $S=Y_{r}$, where $Y_{0}=\mathbb{P}_{2}$ and $Y_{i}$ is obtained from $Y_{i-1}$ by blowing up a point such that each $Y_{i}$ carries an action of an algebraic 2-torus with finitely many fixpoints satisfying conditions (1) and (2) of section 3.1. This just means that each $Y_{i}$ is obtained from $Y_{i-1}$ by blowing up a fixpoint. We can deform $S$ to $\mathbb{P}_{2}\left(x_{1}, \ldots x_{r}\right)$, but under this deformation the good ample cone $C_{S}^{g}$ of $S$ will in general correspond to a proper subcone $C^{g}$ of $C_{\mathbb{P}_{2}\left(x_{1}, \ldots x_{r}\right)}$. Note that $C^{g}$ contains a neighbourhood of the hyperplane class $H$. It is enough to prove that there is such an algorithm computing the Donaldson invariants of $S$ for all generic polarisations in $C_{S}^{g}$.

Fix $c_{1} \in \operatorname{Pic}(S)$ and $c_{2} \in H^{2}(S, \mathbb{Z})$. Let $N:=4 c_{2}-c_{1}^{2}-3$. Let $H_{1}$ and $H_{2}$ be two good generic polarisations of $S$. Then by lemma 2.9 the set $W_{c_{1}, c_{2}}\left(H_{1}, H_{2}\right)$ is finite and consists only of good walls. Therefore

$$
\Phi_{c_{1}, N}^{S, H_{2}}=\Phi_{c_{1}, N}^{S, H_{1}}+\sum_{\xi \in W_{c_{1}, c_{2}}^{S}\left(H_{1}, H_{2}\right)}(-1)^{e_{\xi, N}} \delta_{\xi, N},
$$

and all the $\delta_{\xi, N}$ can be determined explicitely by applying the Bott residue formula. So it is enough to determine $\Phi_{c_{1}, N}^{S, H_{0}}$ for one good polarisation $H_{0}$. We will denote by $E_{1}, \ldots, E_{r}$ the exceptional divisors of $S$ over $\mathbb{P}_{2}$.

First case $c_{1} \neq 0$. Denote $c_{1}=a H+b_{1} E_{1}+\ldots+b_{r} E_{r}$, with each of $a, b_{1}, \ldots, b_{r}$ lying in $\{0,1\}$. We denote $D_{i}:=a H+b_{1} E_{1}+\ldots b_{i}+E_{i}$

By reordering the $E_{i}$ we can assume $a \neq 0$ or $b_{1} \neq 0$. Let $F:=H-E_{1}$. Then for $1>>\epsilon>>\delta_{2}>>$ $\ldots>>\delta_{r}$ the divisors $H_{i}:=F+\epsilon E_{1}-\left(\delta_{2} E_{2}+\ldots+\delta_{i} E_{i}\right)$ are polarisations on $Y_{i}$ lying in a chamber of type $\left(D_{i}, c_{2}\right)$ related to the chamber of type $\left(D_{i-1}, c_{2}\right)$ of $H_{i-1}:=F+\epsilon E_{1}-\left(\delta_{2} E_{2}+\ldots \delta_{i-1} E_{i-1}\right)$ on $Y_{i-1}$. So the blowup formulas give $\Phi_{D_{i}}^{Y_{i}, H_{i}}\left(\alpha \check{E}_{i}^{j}\right)=\Phi_{D_{i-1}}^{Y_{i-1}, H_{i-1}}\left(\alpha S_{j}(p t)\right)$ if $b_{i}=1$ (resp. $\Phi_{D_{i}}^{Y_{i}, H_{i}}\left(\alpha \check{E}_{i}^{j}\right)=$ $\Phi_{D_{i-1}}^{Y_{i-1}, H_{i-1}}\left(\alpha B_{j}(p t)\right)$ if $\left.b_{i}=0\right)$ for all $\alpha \in A_{N-j}\left(Y_{i-1}\right)$. The proof of theorem 6.1 gives an algorithm for computing $\Phi_{D_{1}}^{Y_{1}, H_{1}}(\alpha)$ for all $\alpha \in A_{*}\left(Y_{1}\right)$. Thus by induction we get the desired algorithm.

Second case $c_{1}=0$. Let $\widehat{S}$ be the blow up of $S$ in a point and let $E$ be the exceptional divisor. By the first case we have an algorithm for computing the Donaldson invariant $\Phi_{E, N}^{\widehat{S}, H_{\epsilon}}$ for a polarisation $H_{\epsilon}=H_{0}-\epsilon E$ on $\widehat{S}$ lying in a related chamber to that of a generic good polarisation $H_{0}$ of $S$. Then the $S O(3)$-blowup formula gives $\Phi_{0}^{S, H_{0}}(\alpha)=\Phi_{E}^{\widehat{S}, H_{\epsilon}}(\check{E} \alpha)$, and the result follows. 


\section{REFERENCES}

[A-B] Atiyah, M. and Bott, R., The moment map and equivariant cohomology, Topology 23 (1985), 1-28.

[B] Bott, R., A residue formula for holomorphic vector fields, J. of Diff. Geometry 1 (1967), 311-330.

[C-L1] Carrell, J.B. and Lieberman, D.I., Holomorphic vector fields and Kaehler manifolds, Invent. Math. 21 (1973), 303-309.

[C-L2] Carrell, J.B. and Lieberman, D.I., Vector fields and Chern numbers Math. Ann. 225 (1977),263-273.

[Do] Donaldson, S.K., Polynomial invariants for smooth four-manifolds, Topology 29 (1990), 257-315.

[E] Ellingsrud, G., A new proof of the irreducability of the punctual Hilbert scheme, preprint.

[E-G] Ellingsrud, G. and Göttsche, L., Variation of moduli spaces and Donaldson invariants under change of polarisation, preprint 1994.

[E-LP-S] Ellingsrud, G., Le Potier, J. and Strømme, S.A., Some Donaldson Invariants of $\mathbb{C P}^{2}$, preprint 1995.

[E-S1] Ellingsrud, G. and Strømme, S.A., On the homology of the Hilbert scheme of points in the plane, Invent. Math. 87 (1987), 343-352.

[E-S2] Ellingsrud, G. and Strømme, S.A., Botts formula and enumerative geometry, to appear in Journal of the AMS.

[Fo] Fogarty, J., Algebraic families on an algebraic surface, Amer. J. Math. 90 (1968), 511-521

[F-M] Friedman, R., Morgan, J., On the diffeomorphism types of certain algebraic surfaces, I, J. Diff. Geom. 27 (1988), 297-369.

[F-Q] Friedman, R., Qin, Z., Flips of moduli spaces and transition formulas for Donaldson polynomial invariants of rational surfaces, preprint.

[F-S] Fintushel, R., Stern, R.J., The blowup formula for Donaldson invariants, preprint 1994.

[Gi] Gieseker, D., On the moduli of vector bundles on an algebraic surface, Ann. Math. 106 (1977), 45-60.

[Gö] Göttsche, L., Change of polarization and Hodge numbers of moduli spaces of torsion free sheaves on surfaces, to appear in Math. Zeitschrift.

[H-P] Hyun, S., Park, J.S. Holomorphic Yang-Mills Theory and Variation of the Donaldson Invariants, hepth/9503036.

[Ko] Kotschick, D., SO(3)-invariants for 4-manifolds with $b^{+}=1$, Proc. London Math. Soc. 63 (1991), 426-448.

[K-L] Kotschick, D., Lisca, P., Instanton Invariants of $\mathbb{C P}^{2}$ via Topology, to appear in Math. Annalen.

[K-M] Kotschick, D., Morgan, J., SO(3)-invariants for 4-manifolds with $b^{+}=1$ II, J. Diff. Geom. 39 (1994), 433-456.

[L] Leness, T. Blow-up formulae for SO(3)-Donaldson invariants, preprint.

[L-Q] Li, W.P., Qin, Z., Lower-degree Donaldson polynomials of rational surfaces, J. Alg. Geom. 2 (1993), 413-442.

[Li] Li, J., Algebraic geometric interpretation of Donaldson's polynomial invariants, J. Diff. Geom. 37 (1993), $417-465$.

[Ma1] Maruyama, M., Moduli of stable sheaves I, J. Math. Kyoto Univ., 17 (1977), 91-126.

[Ma2] Maruyama, M., Moduli of stable sheaves II, J. Math. Kyoto Univ. 18 (1978), 557-614.

[M-W] Matsuki, K., Wentworth, R., Mumford-Thaddeus principle on the moduli space of vector bundles on an algebraic surface, preprint 1994.

[Mo] Morgan, J., Comparison of the Donaldson polynomial invariants with their algebro-geometric analogues, Topology 32 (1993), 449-488.

[OG1] O'Grady, K.G., Algebro-geometric analogues of Donaldson's polynomials, Invent. Math. 107 (1992), 351-395.

[O] Ozsváth, P., Some Blowup formulas for SU(2) Donaldson Polynomials, J. Diff. Geom. 40 (1994), 411-447.

[Q1] Qin, Z., Equivalence classes of polarizations and moduli spaces of sheaves, J. Diff. Geom. 37 (1993), $397-413$.

[Q2] Qin, Z., Moduli of stable sheaves on ruled surfaces and their Picard groups, J. reine angew. Math. 433 (1992), 201-219.

[Q3] Qin, Z., Complex structures on certain differentiable 4-manifolds, Topology 32 (1993), 551-566.

[T] Taubes, C.H., in preparation. 
Mathematical Institute, University of Oslo, P. O. Box 1053, N-0316 Oslo, Norway

E-mail address: ellingsr@math.uio.no

Max-Planck-Institut für Mathematik, Gottfried-Claren-Strasse 26, D-53225 Bonn, Germany

E-mail address: lothar@mpim-bonn.mpg.de 